\title{
Medicinal Flowers. XXX.1) Eight New Glycosides, Everlastosides F-M, from the Flowers of Helichrysum arenarium
}

\author{
Toshio Morikawa, ${ }^{a}$ Li-Bo Wang, ${ }^{b, c}$ Kiyofumi Ninomiya, ${ }^{a}$ Seikou Nakamura, ${ }^{b}$ Hisashi Matsuda, ${ }^{b}$ \\ Osamu Muraoka, ${ }^{a}$ Li-Jun Wu, ${ }^{c}$ and Masayuki YoshiKanA ${ }^{*, a, b}$ \\ ${ }^{a}$ Pharmaceutical Research and Technology Institute, Kinki University; 3-4-1 Kowakae, Higashi-osaka, Osaka 577-8502, \\ Japan: ${ }^{b}$ Kyoto Pharmaceutical University; Misasagi, Yamashina-ku, Kyoto 607-8412, Japan: and ${ }^{c}$ Faculty of Natural \\ Medicines, Shenyang Pharmaceutical University; 103 Wenhua Rd., Shenyang 110016, People's Republic of China. \\ Received April 24, 2009; accepted May 26, 2009; published online May 28, 2009
}

\begin{abstract}
Eight new glycosides, everlastosides F (1), G (2), H (3), I (4), J (5), K (6), L (7), and M (8), were isolated from the methanolic extract of the flowers of Helichrysum arenarium. Their structures were elucidated on the basis of chemical and physicochemical evidence.
\end{abstract}

Key words Helichrysum arenarium; everlastoside; medicinal flower; Asteraceae; everlasting

During the course of our studies on medicinal flowers, ${ }^{1-18)}$ we found that the methanolic extract of the flowers of $\mathrm{He}$ lichrysum arenarium L. MoENCH (Asteraceae, Everlasting in English) was found to inhibit on tumor necrosis factor- $\alpha$ (TNF- $\alpha$ )-induced cytotoxicity in L929 cells. ${ }^{17)}$ From the methanolic extract, nine glycosides, arenariumosides I$\mathrm{IV}^{17)}$ and everlastosides $\mathrm{A}-\mathrm{E},{ }^{18)}$ were isolated together with 46 known compounds. As a continuing study on the constituents from $H$. arenarium, we additionally isolated eight new glycosides named everlastosides F (1), G (2), H (3), I (4), J (5), K (6), L (7), and M (8) together with 7- $O$ - $(\beta$-D-glucopyranosyloxy)-5-hydroxy-1(3H)-isobenzofuranone $(9)$ and licoagroside B (10). This paper deals with the isolation and structure elucidation of $\mathbf{1}-\mathbf{8}$.

The methanolic extract from the dried flowers of $H$. arenarium (19.8\% from the dried flowers) was partitioned into an EtOAc- $\mathrm{H}_{2} \mathrm{O}(1: 1, \mathrm{v} / \mathrm{v})$ mixture to furnish an EtOAc-soluble fraction $(7.6 \%)$ and anaqueous phase. The aqueous phase was subjected to Diaion HP-20 column chromatography $\left(\mathrm{H}_{2} \mathrm{O} \rightarrow \mathrm{MeOH}\right)$ to give $\mathrm{H}_{2} \mathrm{O}$ - and $\mathrm{MeOH}$-eluted fractions (8.6\% and $3.2 \%$, respectively), which was described previously. ${ }^{17)}$ From the $\mathrm{MeOH}$-eluted fraction, $1(0.0075 \%), 2$ $(0.0041 \%), 3(0.0025 \%), 4(0.0080 \%), 5(0.0012 \%), 6$ $(0.024 \%), 7(0.0018 \%)$, and $8(0.093 \%)$ were purified together with 7-O-( $\beta$-D-glucopyranosyloxy)-5-hydroxy-1(3H)isobenzofuranone ${ }^{19)}(\mathbf{9}, 0.039 \%)$ and licoagroside $\mathrm{B}^{20)}(\mathbf{1 0}$, $0.0063 \%$ ) using normal- and reversed-phase silica gel chromatographies and finally HPLC.

Structures of Everlastosides F (1), G (2), and $\mathrm{H}$ (3) Everlastoside F (1) was obtained as a white powder with negative optical rotation $\left([\alpha]_{\mathrm{D}}^{28}-42.5^{\circ}\right.$ in $\left.\mathrm{MeOH}\right)$. In the $\mathrm{UV}$ spectrum of 1 , absorption maximum was observed at 219 $(\log \varepsilon 4.42) \mathrm{nm}$. The IR spectrum of 1 showed absorption bands at 1718 and $1655 \mathrm{~cm}^{-1}$ assignable to ester carbonyl and olefin functions and strong absorption bands at 3550 and $1075 \mathrm{~cm}^{-1}$ suggestive of a glycoside moiety. The positive-ion fast atom bombardment (FAB)-MS of 1 showed a quasimolecular ion peak at $\mathrm{m} / \mathrm{z} 447(\mathrm{M}+\mathrm{Na})^{+}$. The molecular formula, $\mathrm{C}_{17} \mathrm{H}_{28} \mathrm{O}_{12}$, of 1 was determined by high resolution FAB-MS measurement. Alkaline hydrolysis of 1 with $10 \%$ aqueous $\mathrm{KOH}-50 \%$ aqueous 1,4 -dioxane $(1: 1, \mathrm{v} / \mathrm{v})$ gave a residue together with angelic acid, which was identified by HPLC analysis of its $p$-nitrobenzyl derivative..$^{2,4,5,7,8,10,13)}$ The residue was successively treated with $1.0 \mathrm{M} \mathrm{HCl}$ liberated D-glucose, which was identified by HPLC using an optical rotation detector. ${ }^{3,11,12,17,18)}$ The ${ }^{1} \mathrm{H}$ - and ${ }^{13} \mathrm{C}-\mathrm{NMR}$ ( $\mathrm{CD}_{3} \mathrm{OD}$, Table 1) spectra of 1, which were assigned by various NMR experiments ${ }^{21)}$ showed signals assignable to two methyls $[\delta 1.92$ $\left(3 \mathrm{H}\right.$, br s, $\left.\left.5-\mathrm{H}_{3}\right), 2.01\left(3 \mathrm{H}, \mathrm{dd}, J=1.2,7.3 \mathrm{~Hz}, 4-\mathrm{H}_{3}\right)\right]$, a trisubstituted olefin $\left[\delta 6.22(1 \mathrm{H}, \mathrm{m}, 3-\mathrm{H}), \delta_{\mathrm{C}} 128.5(2-\mathrm{C})\right.$, 141.3 (3-C)] together with two $\beta$-D-glucopyranosyl parts $[\delta$ $\left.4.33\left(1 \mathrm{H}, \mathrm{d}, J=7.9 \mathrm{~Hz}, 1^{\prime \prime}-\mathrm{H}\right), 5.55\left(1 \mathrm{H}, \mathrm{d}, J=7.9 \mathrm{~Hz}, 1^{\prime}-\mathrm{H}\right)\right]$. The connectivities of glycopyranosyl parts were determined by a heteronuclear multiple-bond correlations (HMBC) experiment on 1. Namely, long-range correlations were observed between the terminal-Glc-1-proton ( 1 "-H) and the inner-Glc-6-carbon $\left(\delta_{\mathrm{C}} 69.5,6^{\prime}-\mathrm{C}\right)$ and between the innerGlc-1-proton $\left(1^{\prime}-\mathrm{H}\right)$ and the angeloyl ester carbonyl carbon $\left(\delta_{\mathrm{C}} 167.7,1-\mathrm{C}\right)$ (Fig. 1). On the basis of the above-mentioned evidence, the structure of everlastoside $F$ was determined to be angeloyl $\beta$-D-glucopyranosyl- $(1 \rightarrow 6)-\beta$-D-glucopyranoside (1).

Everlastoside $\mathrm{G}(\mathbf{2}), \mathrm{C}_{19} \mathrm{H}_{32} \mathrm{O}_{13}$, was observed as a white powder with negative optical rotation $\left([\alpha]_{\mathrm{D}}^{24}-30.3^{\circ}\right.$ in $\mathrm{MeOH})$. The alkaline hydrolysis of $\mathbf{2}$, angelic acid was identified as its acyl group by HPLC analysis of its $p$-nitrobenzyl derivative. The residue was successively treated with $1.0 \mathrm{M}$ $\mathrm{HCl}$ liberated $\mathrm{D}$-apiose ${ }^{18,22,23)}$ and D-glucose, which were identified by HPLC using an optical rotation detector. The ${ }^{1} \mathrm{H}$ - and ${ }^{13} \mathrm{C}$-NMR $\left(\mathrm{CD}_{3} \mathrm{OD}\right.$, Table 1$)$ spectra of $\mathbf{2}$ showed signals assignable to two methylenes and a methine bearing an oxygen function $\left[\delta 3.70\left(2 \mathrm{H}, \mathrm{m}, 3-\mathrm{H}_{2}\right), 4.01(1 \mathrm{H}, \mathrm{m}, 2-\mathrm{H})\right.$, $4.26,4.31\left(1 \mathrm{H}\right.$ each, both dd, $\left.\left.J=5.2,11.9 \mathrm{~Hz}, 1-\mathrm{H}_{2}\right)\right]$ and an angeloyl moiety $\left[\delta 1.90\left(3 \mathrm{H}\right.\right.$, br s, $\left.5^{\prime}-\mathrm{H}_{3}\right), 1.98(3 \mathrm{H}$, dd, $\left.\left.J=1.5,7.3 \mathrm{~Hz}, 4^{\prime}-\mathrm{H}_{3}\right), 6.14\left(1 \mathrm{H}, \mathrm{m}, 3^{\prime}-\mathrm{H}\right)\right]$ together with a $\beta$ D-glucopyranosyl and a $\beta$-D-apiofuranosyl parts $[\delta 4.43(1 \mathrm{H}$, d, $\left.\left.J=8.0 \mathrm{~Hz}, 1^{\prime \prime}-\mathrm{H}\right), 5.00\left(1 \mathrm{H}, \mathrm{d}, J=2.5 \mathrm{~Hz}, 1^{\prime \prime \prime}-\mathrm{H}\right)\right]$. Finally, the connectivities of the acyl group and the glycosyl linkages in 2 were elucidated on the basis of HMBC experiment, which showed long-range correlations between the following proton and carbon pairs as shown in Fig. 1: the 1-protons and the angeloyl ester carbonyl carbon $\left(\delta_{\mathrm{C}} 169.1,1^{\prime}-\mathrm{C}\right)$, the Glc-1-proton $\left(1^{\prime \prime}-\mathrm{H}\right)$ and the 2-carbon $\left(\delta_{\mathrm{C}} 79.9\right)$, and the Api-1-proton $\left(1^{\prime \prime \prime}-\mathrm{H}\right)$ and the Glc-6-carbon $\left(\delta_{\mathrm{C}} 68.9,6^{\prime \prime}-\mathrm{C}\right)$. Consequently, everlastoside $\mathrm{G}$ was determined to be 1-Oangeloylglycerol $2-O$ - $\beta$-D-apiofuranosyl- $(1 \rightarrow 6)-\beta$-D-glucopy- 

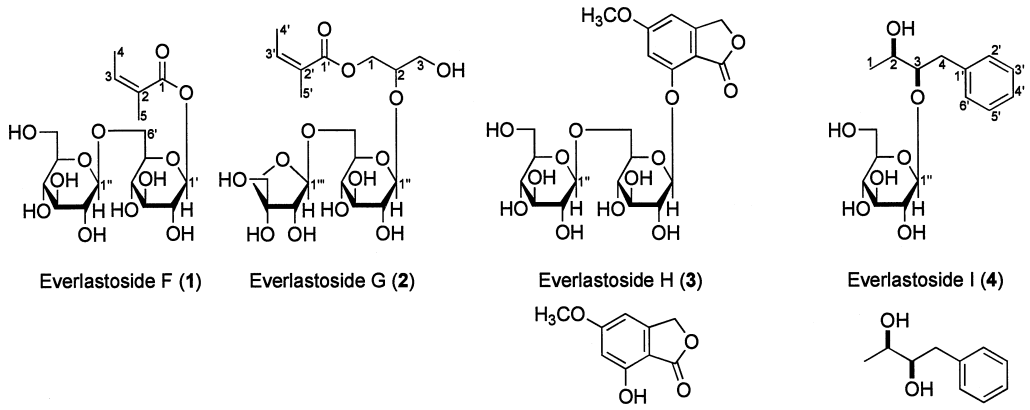

Everlastoside I (4)
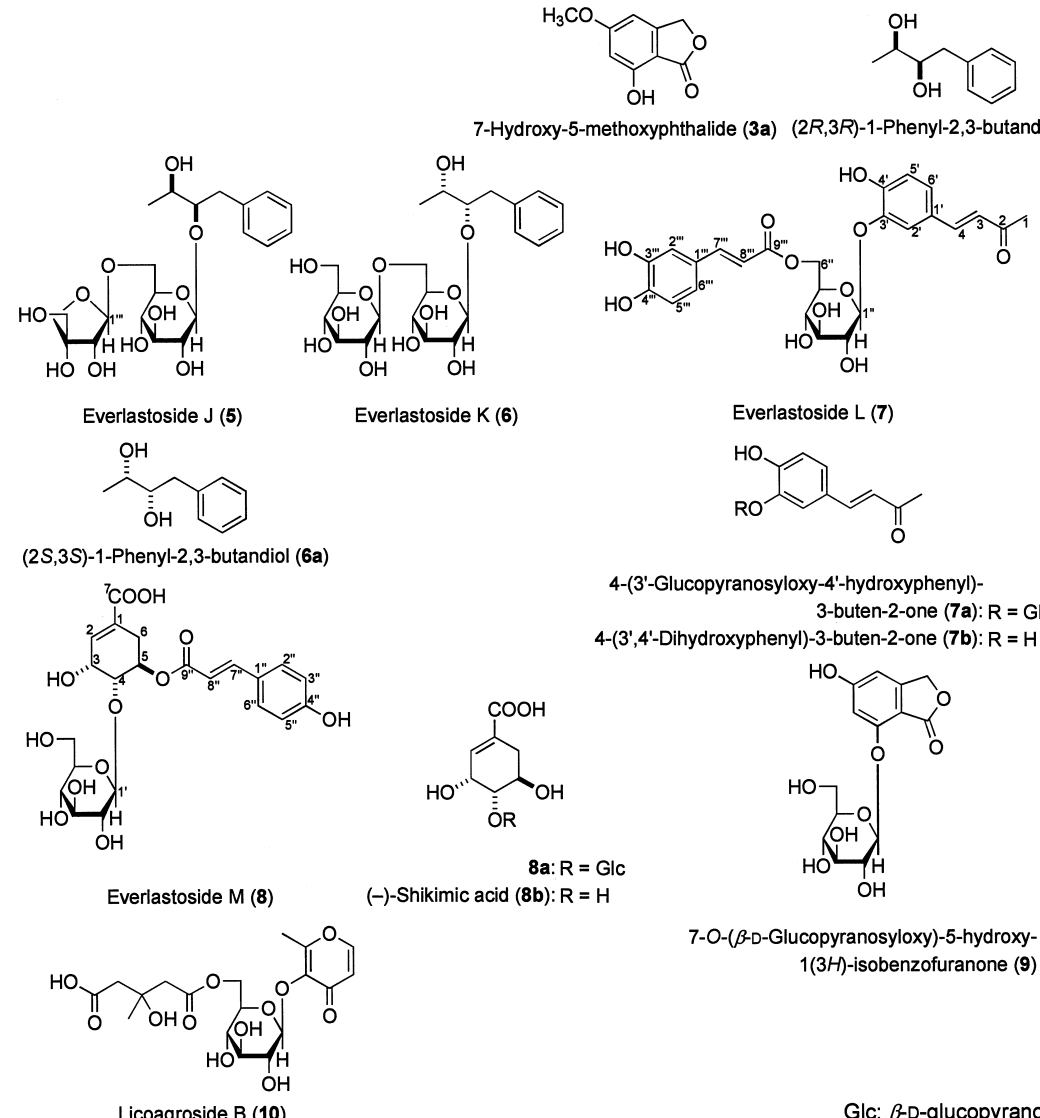

$(-)-$ Shikimic acid $(8 b): R=H$

4-(3'-Glucopyranosyloxy-4'-hydroxyphenyl)3-buten-2-one (7a): $R=\mathrm{Glc}$ 4-(3',4'-Dihydroxyphenyl)-3-buten-2-one (7b): $\mathrm{R}=\mathrm{H}$

$\mathrm{HO}$

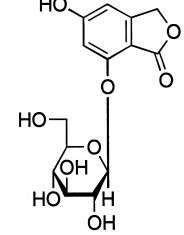

7-O-( $\beta$-D-Glucopyranosyloxy)-5-hydroxy-

$1(3 H)$-isobenzofuranone (9)

Licoagroside B (10)

GlC: $\beta$-D-glucopyranosyl

Chart 1

ranoside (2).

Everlastoside $\mathrm{H}(\mathbf{3})$ was also isolated as a white powder with negative optical rotation $\left([\alpha]_{\mathrm{D}}^{28}-35.2^{\circ}, \mathrm{MeOH}\right)$. Its molecular formula $\mathrm{C}_{21} \mathrm{H}_{28} \mathrm{O}_{14}$ was determined from the positiveion FAB-MS and by high resolution FAB-MS measurements. Acid hydrolysis of $\mathbf{3}$ with $1.0 \mathrm{M} \mathrm{HCl}$ liberated D-glucose, which was identified by HPLC analysis. Enzymatic hydrolysis of 3 with cellulase gave 7-hydroxy-5-methoxyphthalide ${ }^{24,25)}(\mathbf{3 a})$ as an aglycone. The proton and carbon signals in the ${ }^{1} \mathrm{H}$ - and ${ }^{13} \mathrm{C}-\mathrm{NMR}\left(\mathrm{CD}_{3} \mathrm{OD}\right.$, Table 2$)$ spectra of $\mathbf{3}$ were similar to those of $\mathbf{3 a}$, except for the signals due to the oligoglycosyl moiety $\left[\delta 3.91\left(3 \mathrm{H}, \mathrm{s},-\mathrm{OCH}_{3}\right), 4.33(1 \mathrm{H}\right.$, d, $\left.J=7.6 \mathrm{~Hz}, 1^{\prime \prime}-\mathrm{H}\right), 5.08\left(1 \mathrm{H}, \mathrm{d}, J=7.7 \mathrm{~Hz}, 1^{\prime}-\mathrm{H}\right), 5.25(2 \mathrm{H}$, s, $\left.3-\mathrm{H}_{2}\right), 6.77(1 \mathrm{H}$, br s, $\left.4-\mathrm{H}), 6.83(1 \mathrm{H}, \mathrm{d}, J=1.5 \mathrm{~Hz}, 6-\mathrm{H})\right]$. The position of the methoxy group in $\mathbf{3}$ was also clarified by nuclear Overhauser effect spectroscopy (NOESY) experiment, which showed NOE correlations between the methoxy proton and the both aromatic protons $(4-\mathrm{H}$ and $6-\mathrm{H})$. The connectivities of the glycosyl linkages in $\mathbf{3}$ were elucidated on the basis of HMBC experiment, which showed long-range correlations were observed between the terminal-Glc-1-proton $\left(1^{\prime \prime}-\mathrm{H}\right)$ and the inner-Glc-6-carbon $\left(\delta_{\mathrm{C}} 70.5,6^{\prime}-\mathrm{C}\right)$, and between the inner-Glc-1-proton $\left(1^{\prime}-\mathrm{H}\right)$ and the 7 -carbon $\left(\delta_{\mathrm{C}}\right.$ 158.2). Thus, the structure of everlastoside $\mathrm{H}$ was constructed as 7 -hydroxy-5-methoxyphthalide $7-O-\beta$-D-glucopyranosyl $(1 \rightarrow 6)$ - $\beta$-D-glucopyranoside (3).

Structures of Everlastosides I (4), J (5), and K (6) Everlastoside I (4) was obtained as a white powder with negative optical rotation $\left([\alpha]_{\mathrm{D}}^{23}-32.0^{\circ}\right.$ in $\left.\mathrm{MeOH}\right)$. The molecular formula, $\mathrm{C}_{16} \mathrm{H}_{24} \mathrm{O}_{7}$, of $\mathbf{4}$ was determined by a quasimolecular ion peak in the positive-ion FAB-MS and the high resolution FAB-MS measurements. On the other hand, everlastoside $\mathrm{J}(\mathbf{5}), \mathrm{C}_{21} \mathrm{H}_{32} \mathrm{O}_{11}$, was also obtained as a white powder with negative optical rotation $\left([\alpha]_{\mathrm{D}}^{26}-89.3^{\circ}\right.$ in $\left.\mathrm{MeOH}\right)$. The IR spectra of $\mathbf{4}$ and 5 showed similar absorption bands (4: $3568,1509,1458,1375$, and $1071 \mathrm{~cm}^{-1}$; 5: 3568,1508 , 1458,1341 , and $1071 \mathrm{~cm}^{-1}$ ) ascribable to hydroxyl and ether functions and aromatic ring. Treatment of $\mathbf{4}$ and $\mathbf{5}$ with $1.0 \mathrm{M}$ $\mathrm{HCl}$ liberated $(2 R, 3 R)$-1-phenyl-2,3-butanediol ${ }^{26)}(\mathbf{4 a})$ as the common aglycone together with D-glucose (from $\mathbf{4}$ and 5) and D-apiose (from 5), which were identified by HPLC analysis. The ${ }^{1} \mathrm{H}$ - and ${ }^{13} \mathrm{C}-\mathrm{NMR}\left(\mathrm{CD}_{3} \mathrm{OD}\right.$, Table 3$)$ spectra of 4 showed signals assignable to an aglycon part $\{$ a methyl $[\delta$ $\left.1.30\left(3 \mathrm{H}, \mathrm{d}, J=6.1 \mathrm{~Hz}, 1-\mathrm{H}_{3}\right)\right]$, a methylene $[\delta 2.71(1 \mathrm{H}, \mathrm{dd}$, 
Table 1. ${ }^{1} \mathrm{H}$ - and ${ }^{13} \mathrm{C}-\mathrm{NMR}$ Data of Everlastosides F (1) and G (2)

\begin{tabular}{|c|c|c|c|c|c|}
\hline \multirow{2}{*}{ Position } & \multicolumn{2}{|c|}{1} & \multirow{2}{*}{ Position } & \multicolumn{2}{|c|}{2} \\
\hline & $\delta_{\mathrm{H}}(J \mathrm{~Hz})$ & $\delta_{\mathrm{C}}$ & & $\delta_{\mathrm{H}}(J \mathrm{~Hz})$ & $\delta_{\mathrm{C}}$ \\
\hline \multirow[t]{2}{*}{1} & & 167.7 & 1 & $4.26(1 \mathrm{H}, \mathrm{dd}, 5.2,11.9)$ & 64.6 \\
\hline & & & & $4.31(1 \mathrm{H}, \mathrm{dd}, 5.2,11.9)$ & \\
\hline 2 & & 128.5 & 2 & $4.01(1 \mathrm{H}, \mathrm{m})$ & 79.9 \\
\hline 3 & $6.22(1 \mathrm{H}, \mathrm{m})$ & 141.3 & 3 & $3.70(2 \mathrm{H}, \mathrm{m})$ & 63.5 \\
\hline 4 & $2.01(3 \mathrm{H}, \mathrm{dd}, 1.2,7.3)$ & 16.1 & $1^{\prime}$ & & 169.1 \\
\hline \multirow[t]{4}{*}{5} & $1.92(3 \mathrm{H}, \mathrm{brs})$ & 20.6 & $2^{\prime}$ & & 128.9 \\
\hline & & & $3^{\prime}$ & $6.14(1 \mathrm{H}, \mathrm{m})$ & 139.6 \\
\hline & & & $4^{\prime}$ & $1.98(3 \mathrm{H}, \mathrm{dd}, 1.5,7.3)$ & 16.1 \\
\hline & & & $5^{\prime}$ & $1.90(3 \mathrm{H}, \mathrm{brs})$ & 20.8 \\
\hline $1^{\prime}$ & $5.55(1 \mathrm{H}, \mathrm{d}, 7.9)$ & 95.5 & $1^{\prime \prime}$ & $4.43(1 \mathrm{H}, \mathrm{d}, 8.0)$ & 104.3 \\
\hline $2^{\prime}$ & $3.34(1 \mathrm{H}, \mathrm{m})$ & 73.9 & $2^{\prime \prime}$ & $3.20(1 \mathrm{H}, \mathrm{m})$ & 75.0 \\
\hline $3^{\prime}$ & $3.57(1 \mathrm{H}, \mathrm{m})$ & 77.7 & $3^{\prime \prime}$ & $3.34(1 \mathrm{H}, \mathrm{m})$ & 77.8 \\
\hline $4^{\prime}$ & $3.45(1 \mathrm{H}, \mathrm{m})$ & 70.9 & $4^{\prime \prime}$ & $3.24(1 \mathrm{H}, \mathrm{m})$ & 71.8 \\
\hline $5^{\prime}$ & $3.36(1 \mathrm{H}, \mathrm{m})$ & 77.9 & $5^{\prime \prime}$ & $3.43(1 \mathrm{H}, \mathrm{m})$ & 76.9 \\
\hline \multirow[t]{2}{*}{$6^{\prime}$} & $3.78(1 \mathrm{H}, \mathrm{dd}, 5.5,11.6)$ & 69.5 & $6^{\prime \prime}$ & $3.52(1 \mathrm{H}, \mathrm{m})$ & 68.9 \\
\hline & $4.18(1 \mathrm{H}, \mathrm{brd}, c a .12)$ & & & $3.98(1 \mathrm{H}, \mathrm{m})$ & \\
\hline $1^{\prime \prime}$ & $4.33(1 \mathrm{H}, \mathrm{d}, 7.9)$ & 104.5 & $1^{\prime \prime \prime}$ & $5.00(1 \mathrm{H}, \mathrm{d}, 2.5)$ & 111.0 \\
\hline $2^{\prime \prime}$ & $3.23(1 \mathrm{H}, \mathrm{m})$ & 75.0 & $2^{\prime \prime \prime}$ & $3.90(1 \mathrm{H}, \mathrm{d}, 2.5)$ & 77.9 \\
\hline $3^{\prime \prime}$ & $3.45(1 \mathrm{H}, \mathrm{m})$ & 77.9 & $3^{\prime \prime \prime}$ & & 80.5 \\
\hline \multirow[t]{2}{*}{$4^{\prime \prime}$} & $3.30(1 \mathrm{H}, \mathrm{m})$ & 71.4 & $4^{\prime \prime \prime}$ & $3.20(1 \mathrm{H}, \mathrm{m})$ & 74.9 \\
\hline & & & & $3.98(1 \mathrm{H}, \mathrm{m})$ & \\
\hline $5^{\prime \prime}$ & $3.36(1 \mathrm{H}, \mathrm{m})$ & 77.9 & $5^{\prime \prime \prime}$ & $3.54(2 \mathrm{H}, \mathrm{m})$ & 65.5 \\
\hline \multirow[t]{2}{*}{$6^{\prime \prime}$} & $3.68(1 \mathrm{H}, \mathrm{dd}, 5.5,11.6)$ & 62.6 & $6^{\prime \prime \prime}$ & & \\
\hline & $3.85(1 \mathrm{H}, \mathrm{br} \mathrm{d}, \mathrm{ca} .12)$ & & & & \\
\hline
\end{tabular}
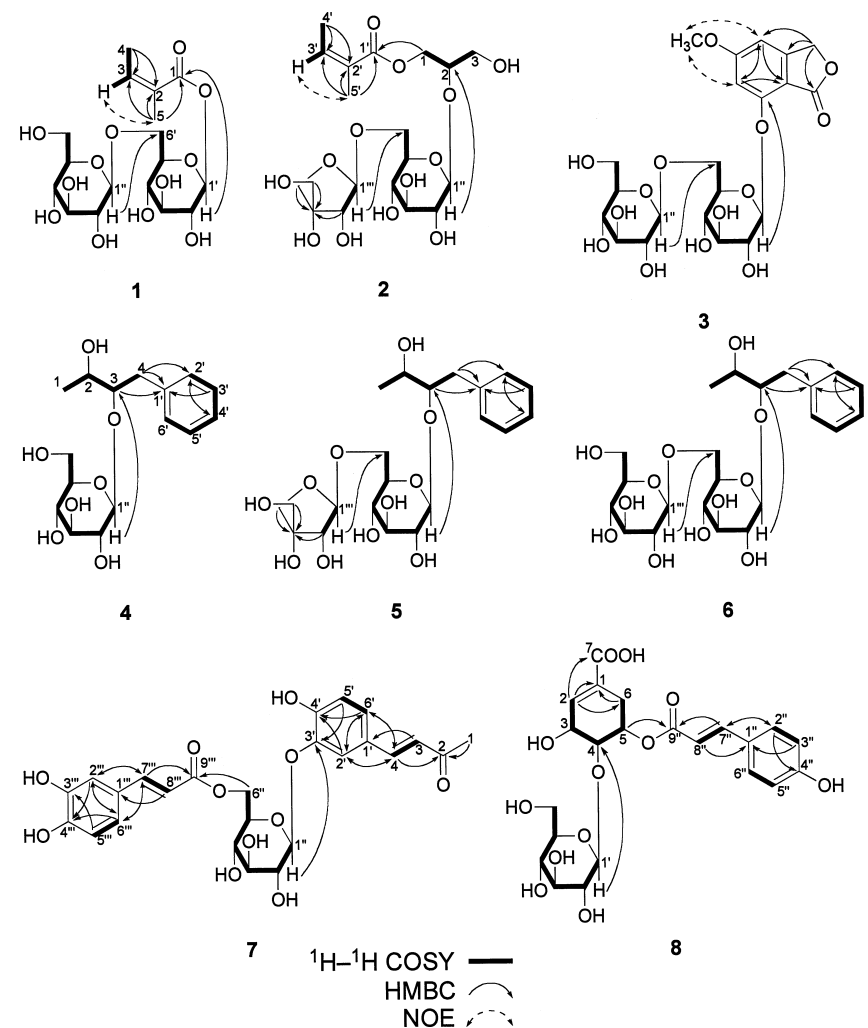

Fig. 1. ${ }^{1} \mathrm{H}-{ }^{1} \mathrm{H}$ COSY, HMBC, and NOE Correlations of $\mathbf{1}-\mathbf{8}$

$\left.J=8.6,14.4 \mathrm{~Hz}), 2.93(1 \mathrm{H}, \mathrm{dd}, J=4.0,14.4 \mathrm{~Hz}), 4-\mathrm{H}_{2}\right]$, two methines bearing an oxygen function $[\delta 3.72(1 \mathrm{H}, \mathrm{m}, 2-\mathrm{H})$, $3.74(1 \mathrm{H}, \mathrm{m}, 3-\mathrm{H})]$, and a monosubstituted benzene ring $[\delta$ $\left.\left.7.15(1 \mathrm{H}, \mathrm{m}), 7.25(4 \mathrm{H}, \mathrm{m}), 2^{\prime}-6^{\prime}-\mathrm{H}\right]\right\}$ together with a $\beta$ glucopyranosyl moiety $\left[\delta 4.41\left(1 \mathrm{H}, \mathrm{d}, J=7.9 \mathrm{~Hz}, 1^{\prime \prime}-\mathrm{H}\right)\right]$. The
Table 2. ${ }^{1} \mathrm{H}$ - and ${ }^{13} \mathrm{C}-\mathrm{NMR}$ Data of Everlastoside H (3)

\begin{tabular}{|c|c|c|}
\hline \multirow{2}{*}{ Position } & \multicolumn{2}{|c|}{3} \\
\hline & $\delta_{\mathrm{H}}(J \mathrm{~Hz})$ & $\delta_{\mathrm{C}}$ \\
\hline 1 & & 172.0 \\
\hline 3 & $5.25(2 \mathrm{H}, \mathrm{s})$ & 70.6 \\
\hline 4 & $6.77(1 \mathrm{H}, \mathrm{brs})$ & 101.0 \\
\hline 5 & & 168.6 \\
\hline 6 & $6.83(1 \mathrm{H}, \mathrm{d}, 1.5)$ & 104.0 \\
\hline 7 & & 158.2 \\
\hline 8 & & 107.3 \\
\hline 9 & & 153.5 \\
\hline $5-\mathrm{OMe}$ & $3.91(3 \mathrm{H}, \mathrm{s})$ & 56.8 \\
\hline $1^{\prime}$ & $5.08(1 \mathrm{H}, \mathrm{d}, 7.7)$ & 101.6 \\
\hline $2^{\prime}$ & $3.95(1 \mathrm{H}, \mathrm{m})$ & 74.3 \\
\hline $3^{\prime}$ & $3.83(1 \mathrm{H}, \mathrm{m})$ & 77.3 \\
\hline $4^{\prime}$ & $3.42(1 \mathrm{H}, \mathrm{m})$ & 71.5 \\
\hline $5^{\prime}$ & $3.22(1 \mathrm{H}, \mathrm{m})$ & 78.0 \\
\hline \multirow[t]{2}{*}{$6^{\prime}$} & $3.83(1 \mathrm{H}, \mathrm{m})$ & 70.5 \\
\hline & $4.14(1 \mathrm{H}, \mathrm{brd}, c a .10)$ & \\
\hline $1^{\prime \prime}$ & $4.33(1 \mathrm{H}, \mathrm{d}, 7.6)$ & 105.2 \\
\hline $2^{\prime \prime}$ & $3.22(1 \mathrm{H}, \mathrm{m})$ & 75.0 \\
\hline $3^{\prime \prime}$ & $3.83(1 \mathrm{H}, \mathrm{m})$ & 77.4 \\
\hline $4^{\prime \prime}$ & $3.24(1 \mathrm{H}, \mathrm{m})$ & 71.6 \\
\hline $5^{\prime \prime}$ & $3.28(1 \mathrm{H}, \mathrm{m})$ & 77.9 \\
\hline \multirow[t]{2}{*}{$6^{\prime \prime}$} & $3.65(1 \mathrm{H}, \mathrm{dd}, 6.1,11.9)$ & 62.8 \\
\hline & $3.83(1 \mathrm{H}, \mathrm{m})$ & \\
\hline
\end{tabular}

HMBC experiment on 4, long-range correlations were observed between the Glc-1-proton $\left(1^{\prime \prime}-\mathrm{H}\right)$ and the 3 -carbon $\left(\delta_{\mathrm{C}}\right.$ $80.5)$ as shown in Fig. 1. The proton and carbon signals in the ${ }^{1} \mathrm{H}$ - and ${ }^{13} \mathrm{C}-\mathrm{NMR}$ (Table $3, \mathrm{CD}_{3} \mathrm{OD}$ ) spectra of 5 were superimposable on those of $\mathbf{4}$, except for the signals due to a D-apiofuranosyl part $\left[\delta 5.00\left(1 \mathrm{H}, \mathrm{d}, J=2.1 \mathrm{~Hz}, 1^{\prime \prime \prime}-\mathrm{H}\right)\right]$. The 
Table 3. ${ }^{1} \mathrm{H}$ - and ${ }^{13} \mathrm{C}-\mathrm{NMR}$ Data of Everlastosides I (4), J (5), and K (6)

\begin{tabular}{|c|c|c|c|c|c|c|}
\hline \multirow{2}{*}{ Position } & \multicolumn{2}{|l|}{4} & \multicolumn{2}{|l|}{5} & \multicolumn{2}{|l|}{6} \\
\hline & $\delta_{\mathrm{H}}(J \mathrm{~Hz})$ & $\delta_{\mathrm{C}}$ & $\delta_{\mathrm{H}}(J \mathrm{~Hz})$ & $\delta_{\mathrm{C}}$ & $\delta_{\mathrm{H}}(J \mathrm{~Hz})$ & $\delta_{\mathrm{C}}$ \\
\hline 1 & $1.30(3 \mathrm{H}, \mathrm{d}, 6.1)$ & 18.7 & $1.30(3 \mathrm{H}, \mathrm{d}, 6.2)$ & 18.7 & $1.30(3 \mathrm{H}, \mathrm{d}, 6.4)$ & 18.6 \\
\hline 2 & $3.72(1 \mathrm{H}, \mathrm{m})$ & 77.0 & $3.70(1 \mathrm{H}, \mathrm{m})$ & 77.0 & $3.70(1 \mathrm{H}, \mathrm{m})$ & 77.0 \\
\hline 3 & $3.74(1 \mathrm{H}, \mathrm{m})$ & 80.5 & $3.74(1 \mathrm{H}, \mathrm{brd}, c a .8)$ & 80.4 & $3.75(1 \mathrm{H}, \mathrm{m})$ & 80.3 \\
\hline \multirow[t]{2}{*}{4} & $2.71(1 \mathrm{H}, \mathrm{dd}, 8.6,14.4)$ & 40.0 & $2.72(1 \mathrm{H}, \mathrm{dd}, 8.2,13.7)$ & 40.0 & $2.72(1 \mathrm{H}, \mathrm{dd}, 3.7,13.8)$ & 40.0 \\
\hline & $2.93(1 \mathrm{H}, \mathrm{dd}, 4.0,14.4)$ & & $2.93(1 \mathrm{H}, \mathrm{dd}, 3.5,13.7)$ & & $2.93(1 \mathrm{H}, \mathrm{dd}, 8.8,13.8)$ & \\
\hline $1^{\prime}$ & & 140.7 & & 140.7 & & 140.7 \\
\hline $2^{\prime}, 6^{\prime}$ & $7.25(2 \mathrm{H}, \mathrm{m})$ & 130.5 & $7.26(2 \mathrm{H}, \mathrm{m})$ & 130.5 & $7.25(2 \mathrm{H}, \mathrm{m})$ & 129.5 \\
\hline $3^{\prime}, 5^{\prime}$ & $7.25(2 \mathrm{H}, \mathrm{m})$ & 129.2 & $7.26(2 \mathrm{H}, \mathrm{m})$ & 129.2 & $7.25(2 \mathrm{H}, \mathrm{m})$ & 129.2 \\
\hline $4^{\prime}$ & $7.15(1 \mathrm{H}, \mathrm{m})$ & 127.1 & $7.17(1 \mathrm{H}, \mathrm{m})$ & 127.1 & $7.16(1 \mathrm{H}, \mathrm{m})$ & 127.0 \\
\hline $1^{\prime \prime}$ & $4.41(1 \mathrm{H}, \mathrm{d}, 7.9)$ & 105.4 & $4.38(1 \mathrm{H}, \mathrm{d}, 7.6)$ & 105.2 & $4.42(1 \mathrm{H}, \mathrm{d}, 7.6)$ & 104.9 \\
\hline $2^{\prime \prime}$ & $3.23(1 \mathrm{H}, \mathrm{m})$ & 75.6 & $3.22(1 \mathrm{H}, \mathrm{m})$ & 75.5 & $3.20(1 \mathrm{H}, \mathrm{m})$ & 75.1 \\
\hline $3^{\prime \prime}$ & $3.37(1 \mathrm{H}, \mathrm{m})$ & 78.0 & $3.70(1 \mathrm{H}, \mathrm{m})$ & 77.9 & $3.44(1 \mathrm{H}, \mathrm{m})$ & 77.1 \\
\hline $4^{\prime \prime}$ & $3.35(1 \mathrm{H}, \mathrm{m})$ & 71.6 & $3.22(1 \mathrm{H}, \mathrm{m})$ & 71.8 & $3.35(1 \mathrm{H}, \mathrm{m})$ & 71.6 \\
\hline $5^{\prime \prime}$ & $3.35(1 \mathrm{H}, \mathrm{m})$ & 78.0 & $3.37(1 \mathrm{H}, \mathrm{m})$ & 76.8 & $3.28(1 \mathrm{H}, \mathrm{m})$ & 77.9 \\
\hline \multirow[t]{2}{*}{$6^{\prime \prime}$} & $3.67(1 \mathrm{H}, \mathrm{dd}, 5.5,11.9)$ & 62.8 & $3.59(1 \mathrm{H}, \mathrm{dd}, 6.2,11.0)$ & 68.7 & $3.75(1 \mathrm{H}, \mathrm{m})$ & 69.9 \\
\hline & $3.85(1 \mathrm{H}, \mathrm{dd}, 2.0,11.9)$ & & $3.96(1 \mathrm{H}, \mathrm{dd}, 2.0,11.0)$ & & $4.13(1 \mathrm{H}, \mathrm{dd}, 1.5,11.6)$ & \\
\hline $1^{\prime \prime \prime}$ & & & $5.00(1 \mathrm{H}, \mathrm{d}, 2.1)$ & 111.0 & $4.39(1 \mathrm{H}, \mathrm{d}, 7.6)$ & 105.0 \\
\hline $2^{\prime \prime \prime}$ & & & $3.89(1 \mathrm{H}, \mathrm{d}, 2.1)$ & 77.8 & $3.23(1 \mathrm{H}, \mathrm{m})$ & 75.5 \\
\hline $3^{\prime \prime \prime}$ & & & & 80.5 & $3.44(1 \mathrm{H}, \mathrm{m})$ & 77.8 \\
\hline \multirow[t]{2}{*}{$4^{\prime \prime \prime}$} & & & $3.27(1 \mathrm{H}, \mathrm{m})$ & 75.0 & $3.35(1 \mathrm{H}, \mathrm{m})$ & 71.6 \\
\hline & & & $3.93(1 \mathrm{H}, \mathrm{m})$ & & & \\
\hline $5^{\prime \prime \prime}$ & & & $3.56(2 \mathrm{H}, \mathrm{s})$ & 65.5 & $3.28(1 \mathrm{H}, \mathrm{m})$ & 78.0 \\
\hline \multirow[t]{2}{*}{$6^{\prime \prime \prime}$} & & & & & $3.65(1 \mathrm{H}, \mathrm{dd}, 5.2,11.6)$ & 62.7 \\
\hline & & & & & $3.86(1 \mathrm{H}, \mathrm{m})$ & \\
\hline
\end{tabular}

Measured in $\mathrm{CD}_{3} \mathrm{OD}$.

connectivity of the D-apiofuranosyl part in $\mathbf{5}$ was characterized by $\mathrm{HMBC}$ experiment, which showed long-range correlations between the Api-1-proton (1"'-H) and the Glc-6-carbon $\left(\delta_{\mathrm{C}} 68.7,6^{\prime \prime}-\mathrm{C}\right)$. On the basis of above-mentioned evidence, the structures of everlastosides I and $\mathrm{J}$ were elucidated to be $(2 R, 3 R)-1$-phenyl-2,3-butanediol 3-O- $\beta$-D-glucopyranoside (4) and $(2 R, 3 R)$-1-phenyl-2,3-butanediol 3- $O$ - $\beta$-D-apiofuranosyl-( $(1 \rightarrow 6)-\beta$-D-glucopyranoside $(5)$, respectively.

Everlastoside K (6) was also obtained as a white powder and exhibited a negative optical rotation $\left([\alpha]_{\mathrm{D}}^{25}-34.2^{\circ}\right.$ in $\mathrm{MeOH})$. The IR spectrum of $\mathbf{6}$ showed absorption bands at $3568,1508,1458$, and $1071 \mathrm{~cm}^{-1}$ assignable to hydroxyl and ether functions and aromatic ring. In the positive-ion FABMS of 6, quasimolecular ion peaks were observed at $\mathrm{m} / \mathrm{z} 491$ $(\mathrm{M}+\mathrm{H})^{+}$and $m / z 513(\mathrm{M}+\mathrm{Na})^{+}$, and high-resolution FABMS analysis revealed the molecular formula to be $\mathrm{C}_{22} \mathrm{H}_{34} \mathrm{O}_{12}$. The acid hydrolysis of 6 with $1.0 \mathrm{M} \mathrm{HCl}$ liberated $(2 S, 3 S)-1$ phenyl-2,3-butanediol ${ }^{26)}$ (6a) as an aglycone together with Dglucose, which was identified by HPLC analysis. The proton and carbon signals in the ${ }^{1} \mathrm{H}$ - and ${ }^{13} \mathrm{C}$-NMR (Table 3, $\left.\mathrm{CD}_{3} \mathrm{OD}\right)$ spectra of $\mathbf{6}$ indicated the presence of a methyl $[\delta$ $\left.1.30\left(3 \mathrm{H}, \mathrm{d}, J=6.4 \mathrm{~Hz}, 1-\mathrm{H}_{3}\right)\right]$, a methylene $[\delta 2.72(1 \mathrm{H}$, dd, $3.7,13.8 \mathrm{~Hz}), 2.93(1 \mathrm{H}$, dd, $\left.8.8,13.8 \mathrm{~Hz}), 4-\mathrm{H}_{2}\right]$, two methines bearing an oxygen function $[\delta 3.70(1 \mathrm{H}, \mathrm{m}, 2-\mathrm{H}), 3.75$ $(1 \mathrm{H}, \mathrm{m}, 3-\mathrm{H})]$, and a monosubstituted benzene ring $[\delta 7.16$ $\left.(1 \mathrm{H}, \mathrm{m}), 7.25(4 \mathrm{H}, \mathrm{m}), 2^{\prime}-6^{\prime}-\mathrm{H}\right]$ together with two $\beta$-D-glucopyranosyl moieties $\left[\delta 4.39\left(1 \mathrm{H}, \mathrm{d}, J=7.6 \mathrm{~Hz}, 1^{\prime \prime \prime}-\mathrm{H}\right), 4.42\right.$ $\left.\left(1 \mathrm{H}, \mathrm{d}, J=7.6 \mathrm{~Hz}, 1^{\prime \prime}-\mathrm{H}\right)\right]$. In the HMBC experiment of $\mathbf{6}$, long-range correlations were observed between the following proton and carbon pairs [1"-H and 3-C $\left(\delta_{\mathrm{C}} 80.3\right) ; 1^{\prime \prime \prime}-\mathrm{H}$ and $\left.6^{\prime \prime}-\mathrm{C}\left(\delta_{\mathrm{C}} 69.9\right)\right]$. Consequently, the structure of everlastoside $\mathrm{K}$ was elucidated to be (2S,3S)-1-phenyl-2,3-butanediol 3-O$\beta$-D-glucopyranosyl( $1 \rightarrow 6)$ - $\beta$-D-glucopyranoside (6).
Structures of Everlastosides L (7) and M (8) Everlastoside $\mathrm{L}(7),[\alpha]_{\mathrm{D}}^{22}+34.5^{\circ}(\mathrm{MeOH})$, was obtained as an amorphous powder. The molecular formula, $\mathrm{C}_{19} \mathrm{H}_{32} \mathrm{O}_{13}$, of 7 was determined from the positive-ion FAB-MS and by high resolution positive-ion FAB-MS. In the UV spectrum of 7, absorption maximum was observed at $329(\log \varepsilon 4.07) \mathrm{nm}$. The IR spectrum of 7 showed absorption bands at 3433, $1716,1686,1655,1541,1509,1458$, and $1073 \mathrm{~cm}^{-1}$ assignable to hydroxyl, ester carbonyl, $\alpha, \beta$-unsaturated carbonyl, and ether functions and aromatic ring. The enzymatic hydrolysis of 7 with tannase liberated 4-(3'-glucopyranosyloxy-4' hydroxyphenyl)-3-buten-2-one ${ }^{27)}$ (7a) and caffeic acid. ${ }^{28)}$ The acid hydrolysis of $7 \mathbf{a}$ liberated D-glucose, which was identified by HPLC analysis. The ${ }^{1} \mathrm{H}$ - and ${ }^{13} \mathrm{C}$-NMR (Table 4 , $\left.\mathrm{CD}_{3} \mathrm{OD}\right)$ spectra of 7 showed signals assignable to a methyl $\left[\delta 2.18\left(3 \mathrm{H}, \mathrm{s}, 1-\mathrm{H}_{3}\right)\right]$, a pair of trans-olefin $[\delta 6.56,7.46(1 \mathrm{H}$ each, both $\mathrm{d}, J=16.1 \mathrm{~Hz}, 3,4-\mathrm{H})]$, a trisubstituted benzene ring $\left[\delta 6.88\left(1 \mathrm{H}, \mathrm{d}, J=8.5 \mathrm{~Hz}, 5^{\prime}-\mathrm{H}\right), 7.20(1 \mathrm{H}, \mathrm{dd}, J=1.9\right.$, $\left.\left.8.5 \mathrm{~Hz}, 6^{\prime}-\mathrm{H}\right), 7.39\left(1 \mathrm{H}, \mathrm{d}, J=1.9 \mathrm{~Hz}, 2^{\prime}-\mathrm{H}\right)\right]$, and a $\beta$-D-glucopyranosyl part $\left[\delta 4.89\left(1 \mathrm{H}, \mathrm{d}, J=7.4 \mathrm{~Hz}, 1^{\prime \prime}-\mathrm{H}\right)\right]$ together with a caffeoyl moiety $[\delta 6.21,7.48(1 \mathrm{H}$ each, both $\mathrm{d}$, $\left.J=16.0 \mathrm{~Hz}, 8^{\prime \prime \prime}, 7^{\prime \prime \prime}-\mathrm{H}\right), 6.74\left(1 \mathrm{H}, \mathrm{d}, J=8.3 \mathrm{~Hz}, 5^{\prime \prime \prime}-\mathrm{H}\right), 6.83$ (1H, dd, $\left.J=1.9,8.3 \mathrm{~Hz}, 6^{\prime \prime \prime}-\mathrm{H}\right), 6.94\left(1 \mathrm{H}, \mathrm{d}, J=1.9 \mathrm{~Hz}, 2^{\prime \prime \prime}-\right.$ $\mathrm{H})$ ]. The connectivity of the caffeoyl part in 7 was confirmed by HMBC experiments, in which long-range correlation was observed between the Glc-6-proton $[\delta 4.32(1 \mathrm{H}$, dd, $J=7.4$, $\left.11.8 \mathrm{~Hz}), 4.62(1 \mathrm{H}, \mathrm{dd}, J=1.9,11.8 \mathrm{~Hz}), 6^{\prime \prime}-\mathrm{H}\right]$ and the caffeoryl ester carbonyl carbon $\left(\delta_{\mathrm{C}} 169.0,9^{\prime \prime \prime}-\mathrm{C}\right)$. Thus, the structure of everlastoside L (7) was elucidated to be as shown.

Everlastoside $\mathrm{M}(\mathbf{8}),[\alpha]_{\mathrm{D}}^{26}-80.8^{\circ}(\mathrm{MeOH})$, was also obtained as a white powder. The molecular formula, $\mathrm{C}_{22} \mathrm{H}_{26} \mathrm{O}_{12}$, of 8 was determined from the positive-ion FAB-MS and by high resolution positive-ion FAB-MS. In the UV spectrum of 
Table 4. ${ }^{1} \mathrm{H}$ - and ${ }^{13} \mathrm{C}-\mathrm{NMR}$ Data of Everlastosides L (7) and M (8), and 8a

\begin{tabular}{|c|c|c|c|c|c|c|c|}
\hline \multirow{2}{*}{ Position } & \multicolumn{2}{|l|}{7} & \multirow{2}{*}{ Position } & \multicolumn{2}{|l|}{8} & \multicolumn{2}{|l|}{$8 \mathbf{a}$} \\
\hline & $\delta_{\mathrm{H}}(J \mathrm{~Hz})$ & $\delta_{\mathrm{C}}$ & & $\delta_{\mathrm{H}}(J \mathrm{~Hz})$ & $\delta_{\mathrm{C}}$ & $\delta_{\mathrm{H}}(J \mathrm{~Hz})$ & $\delta_{\mathrm{C}}$ \\
\hline 1 & $2.18(3 \mathrm{H}, \mathrm{s})$ & 26.9 & 1 & & 130.2 & & 133.1 \\
\hline 2 & & 201.5 & 2 & $6.82(1 \mathrm{H}, \mathrm{brs})$ & 139.4 & $6.70(1 \mathrm{H}, \mathrm{d}, 2.0)$ & 136.4 \\
\hline 3 & $6.56(1 \mathrm{H}, \mathrm{d}, 16.1)$ & 125.6 & 3 & $4.53(1 \mathrm{H}$, br s $)$ & 66.7 & $4.50(1 \mathrm{H}, \mathrm{brs})$ & 66.5 \\
\hline 4 & $7.46(1 \mathrm{H}, \mathrm{d}, 16.1)$ & 146.1 & 4 & $4.10(1 \mathrm{H}, \mathrm{dd}, 4.6,4.7)$ & 77.94 & $3.80(1 \mathrm{H}, \mathrm{dd}, 4.1,7.6)$ & 82.0 \\
\hline $1^{\prime}$ & & 127.9 & 5 & $5.58(1 \mathrm{H}, \mathrm{brdd}, c a .5,8)$ & 70.9 & $4.22(1 \mathrm{H}, \mathrm{brdd}, c a .6,8)$ & 67.2 \\
\hline $2^{\prime}$ & $7.39(1 \mathrm{H}, \mathrm{d}, 1.9)$ & 117.9 & 6 & $2.43(1 \mathrm{H}, \mathrm{dd}, 1.4,17.2)$ & 28.0 & $2.23(1 \mathrm{H}, \mathrm{dd}, 5.5,17.9)$ & 32.3 \\
\hline $3^{\prime}$ & & 146.8 & & $2.78(1 \mathrm{H}, \mathrm{brd}, \mathrm{ca} .17)$ & & $2.78(1 \mathrm{H}, \mathrm{brd}, c a .18)$ & \\
\hline $4^{\prime}$ & & 151.5 & & & 169.7 & & 171.4 \\
\hline $5^{\prime}$ & $6.88(1 \mathrm{H}, \mathrm{d}, 8.5)$ & 117.7 & & & & & \\
\hline $6^{\prime}$ & $7.20(1 \mathrm{H}, \mathrm{dd}, 1.9,8.5)$ & 126.5 & & & & & \\
\hline $1^{\prime \prime}$ & $4.89(1 \mathrm{H}, \mathrm{d}, 7.4)$ & 103.4 & $1^{\prime}$ & $4.50(1 \mathrm{H}, \mathrm{d}, 7.6)$ & 105.8 & $4.45(1 \mathrm{H}, \mathrm{d}, 8.2)$ & 104.8 \\
\hline $2^{\prime \prime}$ & $3.55(1 \mathrm{H}, \mathrm{m})$ & 74.8 & $2^{\prime}$ & $3.23(1 \mathrm{H}, \mathrm{dd}, 7.6,8.9)$ & 75.4 & $3.23(1 \mathrm{H}, \mathrm{dd}, 8.2,8.9)$ & 75.2 \\
\hline $3^{\prime \prime}$ & $3.54(1 \mathrm{H}, \mathrm{m})$ & 77.5 & $3^{\prime}$ & $3.38(1 \mathrm{H}, \mathrm{dd}, 8.9,8.9)$ & 77.89 & $3.33(1 \mathrm{H}, \mathrm{m})$ & 77.8 \\
\hline $4^{\prime \prime}$ & $3.40(1 \mathrm{H}, \mathrm{m})$ & 72.1 & $4^{\prime}$ & $3.31(1 \mathrm{H}, \mathrm{m})$ & 71.5 & $3.31(1 \mathrm{H}, \mathrm{m})$ & 71.4 \\
\hline $5^{\prime \prime}$ & $3.79(1 \mathrm{H}, \mathrm{m})$ & 75.9 & $5^{\prime}$ & $3.35(1 \mathrm{H}, \mathrm{m})$ & 78.2 & $3.30(1 \mathrm{H}, \mathrm{m})$ & 78.1 \\
\hline $6^{\prime \prime}$ & $4.32(1 \mathrm{H}, \mathrm{dd}, 7.4,11.8)$ & 64.9 & $6^{\prime}$ & $3.71(1 \mathrm{H}, \mathrm{dd}, 6.2,11.2)$ & 62.9 & $3.66(1 \mathrm{H}, \mathrm{dd}, 4.9,11.7)$ & 62.6 \\
\hline & $4.62(1 \mathrm{H}, \mathrm{dd}, 1.9,11.8)$ & & & $3.90(1 \mathrm{H}, \mathrm{dd}, 2.0,11.2)$ & & $3.87(1 \mathrm{H}, \mathrm{dd}, 1.2,11.7)$ & \\
\hline $1^{\prime \prime \prime}$ & & 127.7 & $1^{\prime \prime}$ & & 126.0 & & \\
\hline $2^{\prime \prime \prime}$ & $6.94(1 \mathrm{H}, \mathrm{d}, 1.9)$ & 115.4 & $2^{\prime \prime}, 6^{\prime \prime}$ & $7.45(2 \mathrm{H}, \mathrm{d}, 8.9)$ & 130.8 & & \\
\hline $3^{\prime \prime \prime}$ & & 146.8 & $3^{\prime \prime}, 5^{\prime \prime}$ & $6.79(2 \mathrm{H}, \mathrm{d}, 8.9)$ & 116.8 & & \\
\hline $4^{\prime \prime \prime}$ & & 149.7 & $4^{\prime \prime}$ & & 161.6 & & \\
\hline $5^{\prime \prime \prime}$ & $6.74(1 \mathrm{H}, \mathrm{d}, 8.3)$ & 116.5 & & & & & \\
\hline $6^{\prime \prime \prime}$ & $6.83(1 \mathrm{H}, \mathrm{dd}, 1.9,8.3)$ & 117.9 & & & & & \\
\hline $7^{\prime \prime \prime}$ & $7.48(1 \mathrm{H}, \mathrm{d}, 16.0)$ & 147.3 & $7^{\prime \prime}$ & $7.61(1 \mathrm{H}, \mathrm{d}, 16.4)$ & 147.2 & & \\
\hline $8^{\prime \prime \prime}$ & $6.21(1 \mathrm{H}, \mathrm{d}, 16.0)$ & 114.8 & $8^{\prime \prime}$ & $6.32(1 \mathrm{H}, \mathrm{d}, 16.4)$ & 114.9 & & \\
\hline $9^{\prime \prime \prime}$ & & 169.0 & $9^{\prime \prime}$ & & 168.4 & & \\
\hline
\end{tabular}

Measured in $\mathrm{CD}_{3} \mathrm{OD}$.

8, absorption maximum was observed at $258(\log \varepsilon 3.87) \mathrm{nm}$. The IR spectrum of $\mathbf{8}$ showed absorption bands at 3451, $1718,1698,1634,1607,1516,1499,1254,1169$, and $1080 \mathrm{~cm}^{-1}$ assignable to hydroxyl, ester carbonyl, $\alpha, \beta$-unsaturated carboxyl, and ether functions and aromatic ring. Alkaline hydrolysis of 8 with $10 \% \mathrm{KOH}-50 \%$ aqueous 1,4 -dioxane $(1: 1, \mathrm{v} / \mathrm{v})$ liberated $\mathbf{8 a}$ together with $p$-coumaric acid. ${ }^{28)}$ The acid hydrolysis of $\mathbf{8 a}$ gave (-)-shikimic acid ${ }^{28)}(\mathbf{8 b})$ together with D-glucose, which was identified by HPLC analysis. The ${ }^{1} \mathrm{H}$ - and ${ }^{13} \mathrm{C}$-NMR (Table $4, \mathrm{CD}_{3} \mathrm{OD}$ ) spectra of $\mathbf{8}$ showed signals assignable to an aglycone moiety $\{\delta[2.43$ $(1 \mathrm{H}, \mathrm{dd}, J=1.4,17.2 \mathrm{~Hz}), 2.78(1 \mathrm{H}$, br d, $J=c a$. $17 \mathrm{~Hz}), 6-$ $\mathrm{H}_{2}$ ], $4.10(1 \mathrm{H}, \mathrm{dd}, J=4.6,4.7 \mathrm{~Hz}, 4-\mathrm{H}), 4.53(1 \mathrm{H}$, br s, $3-\mathrm{H})$, $5.58(1 \mathrm{H}$, br dd, $J=c a .5,8 \mathrm{~Hz}, 5-\mathrm{H}), 6.82(1 \mathrm{H}$, br s, 2-H $)\}$ and a $p$-coumaroyl part $[\delta 6.32,7.61(1 \mathrm{H}$ each, both $\mathrm{d}$, $\left.J=16.4 \mathrm{~Hz}, 8^{\prime \prime}, 7^{\prime \prime}-\mathrm{H}\right), 6.79,7.45$ (2H each, both $\mathrm{d}, J=8.9 \mathrm{~Hz}$, $\left.\left.3^{\prime \prime}, 5^{\prime \prime}, 2^{\prime \prime}, 6^{\prime \prime}-\mathrm{H}\right)\right]$ together with a $\beta$-D-glucopyranosyl part $[\delta$ $\left.4.50\left(1 \mathrm{H}, \mathrm{d}, J=7.6 \mathrm{~Hz}, 1^{\prime}-\mathrm{H}\right)\right]$. The planar structure of 8 was confirmed by ${ }^{1} \mathrm{H}-{ }^{1} \mathrm{H}$ COSY and $\mathrm{HMBC}$ experiments as shown in Fig. 1. Thus, the ${ }^{1} \mathrm{H}-{ }^{1} \mathrm{H}$ COSY experiment on 8 indicated the presence of partial structures written in bold lines, while long-range correlations in the HMBC experiment were observed between the following proton and carbon pairs (5-H and 9"-C; $1^{\prime}-\mathrm{H}$ and 4-C). Furthermore, comparison of the ${ }^{13} \mathrm{C}-\mathrm{NMR}$ spectrum for $\mathbf{8}$ with those for $\mathbf{8 a}$ revealed an acylation shift around the 5-position in $8\left[8\right.$ : $\delta_{\mathrm{C}}$ 77.94 (4-C), 70.9 (5-C), 28.0 (6-C); 8a: $\delta_{\mathrm{C}} 82.0$ (4-C), 67.2 (5-C), $32.3(6-\mathrm{C})]$, so that the connectivity of the $p$-coumaryl moiety in $\mathbf{8}$ was also clarified to be the 5-position of $\mathbf{8 a}$. Thus, the stereostructure of everlastoside $\mathrm{M}$ was determined to be 5-O-p-coumaroyl-(-)-shikimic acid 4- $O-\beta$-D-glucopy- ranoside $(\mathbf{8})$.

\section{Experimental}

The following instruments were used to obtain physical data: specific rotations, Horiba SEPA-300 digital polarimeter $(l=5 \mathrm{~cm})$; UV spectra, Shimadzu UV-1600 spectrometer; IR spectra, Shimadzu FTIR-8100 spectrometer; ${ }^{1} \mathrm{H}-\mathrm{NMR}$ spectra, JEOL JNM-LA500 (500 MHz) and EX-270 (270 MHz) spectrometers; ${ }^{13} \mathrm{C}-\mathrm{NMR}$ spectra, JEOL JNM-LA500 (125 MHz) and EX-270 $(68 \mathrm{MHz})$ spectrometers with tetramethylsilane as an internal standard; FAB-MS and high resolution FAB-MS, JEOL JMS-SX 102A mass spectrometer; HPLC detector, Shimadzu RID-6A refractive index and SPD10A UV-VIS detectors; HPLC column, Cosmosil $5 \mathrm{C}_{18}$-MS-II (Nacalai Tesque, Inc.) $(250 \mathrm{~mm} \times 4.6 \mathrm{~mm}$ i.d. $)$ and $(250 \mathrm{~mm} \times 20 \mathrm{~mm}$ i.d. $)$ columns were used for analytical and preparative purposes, respectively.

The following experimental conditions were used for chromatography: normal-phase silica gel column chromatography (CC), silica gel 60N (Kanto Chemical Co., Ltd., 63-210 mesh, spherical, neutral); reversed-phase silica gel CC, Diaion HP-20 (Nippon Rensui) and Chromatorex ODS DM1020T (Fuji Silysia Chemical, Ltd., 100-200 mesh); normal-phase TLC, precoated TLC plates with silica gel $60 \mathrm{~F}_{254}$ (Merck, $0.25 \mathrm{~mm}$ ); reversed-phase TLC, pre-coated TLC plates with silica gel RP-18 $\mathrm{F}_{254 \mathrm{~S}}$ (Merck, $0.25 \mathrm{~mm}$ ); reversed-phase HPTLC, pre-coated TLC plates with silica gel RP-18 $\mathrm{WF}_{254 \mathrm{~S}}$ (Merck, $0.25 \mathrm{~mm}$ ), detection was achieved by spraying with $1 \%$ $\mathrm{Ce}\left(\mathrm{SO}_{4}\right)_{2}-10 \%$ aqueous $\mathrm{H}_{2} \mathrm{SO}_{4}$, followed by heating.

Plant Material This item was described in a previous report. ${ }^{17)}$

Extraction and Isolation The dried flowers of $H$. arenarium $(3.0 \mathrm{~kg})$ were extracted three times with $\mathrm{MeOH}$ under reflux for $3 \mathrm{~h}$. Evaporation of the solvent under reduced pressure provided a methanolic extract $(593.8 \mathrm{~g}$, $19.8 \%)$. The methanolic extract $(543.8 \mathrm{~g})$ was partitioned between an EtOAc- $\mathrm{H}_{2} \mathrm{O}(1: 1, \mathrm{v} / \mathrm{v})$ mixture, and removal of the solvents in vacuo yielded an EtOAc-soluble fraction $(210.0 \mathrm{~g}, 7.6 \%)$ and an aqueous phase. The aqueous phase was subjected to Diaion HP-20 CC $(3.0 \mathrm{~kg}$, $\left.\mathrm{H}_{2} \mathrm{O} \rightarrow \mathrm{MeOH}\right)$ to give $\mathrm{H}_{2} \mathrm{O}$-eluted fraction $(237.2 \mathrm{~g}, 8.6 \%)$ and $\mathrm{MeOH}-$ eluted fraction $(88.6 \mathrm{~g}, 3.2 \%)$. The $\mathrm{MeOH}$-eluted fraction $(68.6 \mathrm{~g})$ was subjected to normal-phase silica gel $\mathrm{CC}\left[2.5 \mathrm{~kg}, \mathrm{CHCl}_{3}-\mathrm{MeOH}-\mathrm{H}_{2} \mathrm{O}\right.$ $(20: 3: 1 \rightarrow 10: 3: 1 \rightarrow 7: 3: 1$, lower layer $\rightarrow 6: 4: 1, \mathrm{v} / \mathrm{v} / \mathrm{v}) \rightarrow \mathrm{MeOH}]$ to give 12 fractions [Fr. 1 (0.85 g), Fr. 2 (1.20 g), Fr. 3 (0.90 g), Fr. 4 (1.80 g), Fr. 5 
(6.40 g), Fr. 6 (11.00 g), Fr. 7 (5.40 g), Fr. 8 (4.00 g), Fr. 9 (7.10 g), Fr. 10 $(5.80 \mathrm{~g})$, Fr. $11(6.10 \mathrm{~g})$, and Fr. $12(17.10 \mathrm{~g})]$ as reported previously. ${ }^{17,18)}$

Fraction $4(1.80 \mathrm{~g})$ was subjected to reversed-phase silica gel $\mathrm{CC}$ $\left[70 \mathrm{~g}, \mathrm{MeOH}-\mathrm{H}_{2} \mathrm{O}(15: 85 \rightarrow 80: 20, \mathrm{v} / \mathrm{v}) \rightarrow \mathrm{MeOH}\right]$ and HPLC $[\mathrm{MeOH}-$ $\left.\mathrm{H}_{2} \mathrm{O}(20: 80-30: 70, \mathrm{v} / \mathrm{v})\right]$ to give everlastoside $\mathrm{I}(4,22.0 \mathrm{mg}, 0.0055 \%)$ together with everlastoside A $(2.3 \mathrm{mg}, 0.0005 \%)$ and 7-hydroxy-5-methoxyphthalide $7-O-\beta$-D-glucopyranoside $(476.8 \mathrm{mg}, 0.12 \%) .{ }^{17,18)}$ Fraction 5 $(4.00 \mathrm{~g})$ was purified by reversed-phase silica gel $\mathrm{CC}\left[300 \mathrm{~g}, \mathrm{MeOH}-\mathrm{H}_{2} \mathrm{O}\right.$ $(15: 85 \rightarrow 80: 20, \mathrm{v} / \mathrm{v}) \rightarrow \mathrm{MeOH}]$ and HPLC $\left[\mathrm{MeOH}-\mathrm{H}_{2} \mathrm{O}(15: 85-45: 55\right.$, $\mathrm{v} / \mathrm{v})]$ to furnish 7-O-( $\beta$-D-glucopyranosyloxy)-5-hydroxy-1(3H)-isobenzofuranone $(9,93.0 \mathrm{mg}, 0.023 \%)$ and everlastoside I $(4,10.0 \mathrm{mg}, 0.0025 \%)$ together with (2S)-helichrysin $(320.0 \mathrm{mg}, 0.069 \%),(2 R)$-helichrysin $(18.0 \mathrm{mg}$, $0.0045 \%)$, naringenin $7-O$ - $\beta$-D-glucopyranoside $(21.5 \mathrm{mg}, 0.0053 \%)$, apigenin $7-O-\beta$-D-glucopyranoside $(10.0 \mathrm{mg}, 0.0025 \%)$, luteolin $7-O-\beta$-D-glucopyranoside $(10.0 \mathrm{mg}, \quad 0.0025 \%)$, luteolin $3^{\prime}-O-\beta$-D-glucopyranoside (5.3 $\mathrm{mg}, 0.0013 \%)$, kaempferol 3-O- $\beta$-D-glucopyranoside $(2.30 \mathrm{~g}, 0.58 \%$ ), tortoside B $(10.0 \mathrm{mg}, 0.0025 \%)$, 7-hydroxy-5-methoxyphthalide $7-O-\beta$-Dglucopyranoside $(150.0 \mathrm{mg}, 0.037 \%)$, scopolin $(68.0 \mathrm{mg}, 0.017 \%)$, undulatoside A (11.0 mg, 0.0017\%), 4-(3'-glucopyranosyloxy-4'-hydroxyphenyl)-3buten-2-one (7a, $11.3 \mathrm{mg}, 0.0023 \%$ ), syringin $(15.0 \mathrm{mg}, 0.0037 \%)$, dihydrosyringin $(7.0 \mathrm{mg}, 0.0013 \%)$, and eugenyl $\beta$-D-glucopyranoside $(11.8 \mathrm{mg}$, $0.0029 \%) .{ }^{17)}$ Fraction $7(5.40 \mathrm{~g})$ was subjected by reversed-phase silica gel $\mathrm{CC}\left[300 \mathrm{~g}, \mathrm{MeOH}-\mathrm{H}_{2} \mathrm{O} \quad(15: 85 \rightarrow 70: 30, \mathrm{v} / \mathrm{v}) \rightarrow \mathrm{MeOH}\right]$ and HPLC $\left[\mathrm{MeOH}-\mathrm{H}_{2} \mathrm{O}(10: 90-40: 60, \mathrm{v} / \mathrm{v})\right]$ to furnish $9(65.3 \mathrm{mg}, 0.016 \%)$ and everlastoside $\mathrm{J}(\mathbf{5}, 5.0 \mathrm{mg}, 0.0012 \%)$ together with everlastosides B (12.9 mg, 0.0032\%), C (16.9 mg, 0.0042\%), D (6.2 mg, 0.0015\%), and E (23.0 mg, $0.0060 \%),(2 S)$-helichrysin $(223.0 \mathrm{mg}, 0.055 \%),(2 R)$-helichrysin (17.0 mg, $0.0042 \%)$, chalconaringenin $2^{\prime}-O-\beta$-D-glucopyranoside $(305.5 \mathrm{mg}$, $0.076 \%)$, quercetin $3-O$ - $\beta$-D-glucopyranoside $(40.0 \mathrm{mg}, 0.010 \%),(7 R, 8 S)$ dihydrodehydrodiconiferyl alcohol 4-O- $\beta$-D-glucopyranoside $(10.0 \mathrm{mg}$, $0.0025 \%$ ), oricinol $\beta$-D-glucopyranoside (12.2 $\mathrm{mg}, 0.0035 \%$ ), phenethyl alcohol $\beta$-D-xylopyranosyl-(1 $\rightarrow 6)$ - $\beta$-D-glucopyranoside $(6.2 \mathrm{mg}, 0.0015 \%)$, icariside $\mathrm{D}_{1}(90.0 \mathrm{mg}, 0.017 \%)$, and adenosine $(22.0 \mathrm{mg}, 0.0055 \%) .{ }^{17,18}$ Fraction $8(4.00 \mathrm{~g})$ was subjected to reversed-phase silica gel CC $[300 \mathrm{~g}$, $\left.\mathrm{MeOH}-\mathrm{H}_{2} \mathrm{O} \quad(15: 85 \rightarrow 60: 40, \mathrm{v} / \mathrm{v}) \rightarrow \mathrm{MeOH}\right]$ and HPLC $\left[\mathrm{MeOH}-\mathrm{H}_{2} \mathrm{O}\right.$ $(18: 82-40: 60, \mathrm{v} / \mathrm{v})$ or $\left.\mathrm{CH}_{3} \mathrm{CN}-\mathrm{H}_{2} \mathrm{O}(8: 92-11: 89, \mathrm{v} / \mathrm{v})\right]$ to give everlastoside $\mathrm{G}(\mathbf{2}, 16.4 \mathrm{mg}, 0.0041 \%)$ and licoagroside B (10,6.0 mg, 0.0015\%) together with $(2 S)$-helichrysin $(20.0 \mathrm{mg}, \quad 0.0050 \%), \quad(2 R)$-helichrysin $(30.0 \mathrm{mg}, 0.0075 \%)$, helicioside A $(6.0 \mathrm{mg}, 0.0015 \%),(2 R, 3 R)$-dihydrokaempferol $7-O-\beta$-D-glucopyranoside $(40.0 \mathrm{mg}, 0.010 \%)$, chalconaringenin $2^{\prime}-O$ - $\beta$-D-glucopyranoside $(25.0 \mathrm{mg}, 0.0057 \%)$, quercetin $3-O-\beta$-Dglucopyranoside $(70.0 \mathrm{mg}, 0.018 \%$ ), benzyl alcohol $\beta$-D-xylopyranosyl$(1 \rightarrow 6)$ - $\beta$-D-glucopyranoside $(5.0 \mathrm{mg}, 0.0012 \%)$, icariside $\mathrm{F}_{2}(19.0 \mathrm{mg}$, $0.0048 \%)$, and phenethyl alcohol $\beta$-D-xylopyranosyl-( $1 \rightarrow 6)$ - $\beta$-D-glucopyranoside $(7.2 \mathrm{mg}, 0.0018 \%) .{ }^{17)}$ Fraction $9(7.10 \mathrm{~g})$ was subjected by reversedphase silica gel CC $\left[300 \mathrm{~g}, \mathrm{MeOH}-\mathrm{H}_{2} \mathrm{O}(15: 85 \rightarrow 60: 40, \mathrm{v} / \mathrm{v}) \rightarrow \mathrm{MeOH}\right]$ and HPLC $\left[\mathrm{MeOH}-\mathrm{H}_{2} \mathrm{O}(9: 92-35: 65, \mathrm{v} / \mathrm{v})\right]$ to furnish everlastosides $\mathrm{F}(\mathbf{1}$, $30.0 \mathrm{mg}, 0.0075 \%), \mathrm{H}(\mathbf{3}, 10.0 \mathrm{mg}, 0.0025 \%)$, and $\mathrm{K}(\mathbf{6}, 16.0 \mathrm{mg}, 0.0040 \%)$ together with scutellarein $7-O-\beta$-D-glucopyranoside $(7.0 \mathrm{mg}, 0.0017 \%)$, $3^{\prime}$-methylchrysoeriol $\quad 7-O$ - $\beta$-D-glucopyranoside $\quad(13.0 \mathrm{mg}, \quad 0.0033 \%)$, kaempferol 3-O- $\beta$-D-glucopyranoside $(13.0 \mathrm{mg}, 0.0032 \%)$, kaempferol 3 $O$ - $\beta$-D-glucopyranosyl-( $1 \rightarrow 3)$ - $\beta$-D-glucopyranoside $\quad(17.0 \mathrm{mg}, \quad 0.0040 \%)$, quercetin $3-O-\beta$-D-glucopyranoside $(13.3 \mathrm{mg}, 0.0033 \%)$, aureusidin $6-O-\beta$ D-glucopyranoside $(10.0 \mathrm{mg}, 0.0025 \%)$, and benzoyl $\beta$-D-glucopyranosyl$(1 \rightarrow 6)$ - $\beta$-D-glucopyranoside $(36.0 \mathrm{mg}, 0.0090 \%){ }^{17)}$ Fraction $10(5.10 \mathrm{~g})$ was subjected to reversed-phase silica gel $\mathrm{CC}\left[200 \mathrm{~g}, \mathrm{MeOH}-\mathrm{H}_{2} \mathrm{O}\right.$ $(15: 85 \rightarrow 60: 40, \mathrm{v} / \mathrm{v}) \rightarrow \mathrm{MeOH}]$ and $\mathrm{HPLC}\left[\mathrm{MeOH}-\mathrm{H}_{2} \mathrm{O} \quad(15: 85-40: 60\right.$, $\mathrm{v} / \mathrm{v})$, or $\left.\mathrm{MeOH}-\mathrm{CH}_{3} \mathrm{CN}-\mathrm{H}_{2} \mathrm{O}(12: 8: 80, \mathrm{v} / \mathrm{v} / \mathrm{v})\right]$ to give everlastosides $\mathrm{K}(\mathbf{6}, 82.0 \mathrm{mg}, 0.020 \%)$ and $\mathrm{M}(\mathbf{8}, 369.0 \mathrm{mg}, 0.093 \%)$ and $\mathbf{1 0}(19.4 \mathrm{mg}$, $0.0048 \%)$ together with arenariumosides I $(18.0 \mathrm{mg}, 0.0045 \%)$ and II $(15.4 \mathrm{mg}, 0.0038 \%)$, (2S)-helichrysin $(4.0 \mathrm{mg}, 0.0010 \%)$, apigenin $7-O-$ gentiobioside $(16.0 \mathrm{mg}, 0.0040 \%)$, 6-hydroxyluteolin $7-O-\beta$-D-glucopyranoside $(76.0 \mathrm{mg}, 0.019 \%)$, kaempferol $3-O-\beta$-D-glucopyranoside $(4.8 \mathrm{mg}$, $0.0012 \%)$, kaempferol 3 - $O$-gentiobioside $(28.0 \mathrm{mg}, \quad 0.0070 \%)$, rutin $(13.0 \mathrm{mg}, 0.0032 \%)$, maltol $3-O-\beta$-D-apiofuranosyl-( $1 \rightarrow 6)-\beta$-D-glucopyranoside $(70.0 \mathrm{mg}, 0.018 \%)$, and phenethyl alcohol $\beta$-D-glucopyranosyl$(1 \rightarrow 6)$ - $\beta$-D-glucopyranoside $(18.0 \mathrm{mg}, 0.0045 \%) .{ }^{17)}$ Fraction $12(17.10 \mathrm{~g})$ was subjected to reversed-phase silica gel $\mathrm{CC}\left[500 \mathrm{~g}, \mathrm{MeOH}-\mathrm{H}_{2} \mathrm{O}\right.$ $(10: 90 \rightarrow 40: 60, \mathrm{v} / \mathrm{v}) \rightarrow \mathrm{MeOH}]$ and HPLC $\left[\mathrm{MeOH}-\mathrm{H}_{2} \mathrm{O}(30: 70-45: 55\right.$, $\mathrm{v} / \mathrm{v})$ ] to give everlastoside $\mathrm{L}(7,6.9 \mathrm{mg}, 0.0018 \%)$.

Everlastoside F (1): A white powder, $[\alpha]_{\mathrm{D}}^{28}-42.5^{\circ}(c=1.90, \mathrm{MeOH})$. High-resolution positive-ion FAB-MS: Calcd for $\mathrm{C}_{17} \mathrm{H}_{28} \mathrm{O}_{12} \mathrm{Na}(\mathrm{M}+\mathrm{Na})^{+}$ 447.1478; Found 447.1469. UV [ $\left.\lambda_{\max }(\log \varepsilon), \mathrm{MeOH}\right]: 219$ (4.42) nm. IR $\left(\mathrm{KBr}, \mathrm{cm}^{-1}\right): 3550,1718,1655,1075 .{ }^{1} \mathrm{H}-\mathrm{NMR}\left(500 \mathrm{MHz}, \mathrm{CD}_{3} \mathrm{OD}\right) \delta$ : given in Table 1. ${ }^{13} \mathrm{C}-\mathrm{NMR}\left(125 \mathrm{MHz}, \mathrm{CD}_{3} \mathrm{OD}\right) \delta_{\mathrm{C}}$ : given in Table 1. Positive-ion FAB-MS $m / z$ : $447(\mathrm{M}+\mathrm{Na})^{+}$

Everlastoside G (2): A white powder, $[\alpha]_{\mathrm{D}}^{24}-30.3^{\circ}(c=0.61, \mathrm{MeOH})$ High-resolution positive-ion FAB-MS: Calcd for $\mathrm{C}_{19} \mathrm{H}_{32} \mathrm{O}_{13} \mathrm{Na}(\mathrm{M}+\mathrm{Na})^{+}$ 491.1741; Found 491.1745. UV $\left[\lambda_{\max }(\log \varepsilon), \mathrm{MeOH}\right]: 217$ (4.82) nm. IR $\left(\mathrm{KBr}, \mathrm{cm}^{-1}\right): 3568,1718,1686,1508,1458,1066 .{ }^{1} \mathrm{H}-\mathrm{NMR}(500 \mathrm{MHz}$, $\left.\mathrm{CD}_{3} \mathrm{OD}\right) \delta$ : given in Table $1 .{ }^{13} \mathrm{C}-\mathrm{NMR}\left(125 \mathrm{MHz}, \mathrm{CD}_{3} \mathrm{OD}\right) \delta_{\mathrm{C}}$ : given in Table 1. Positive-ion FAB-MS $m / z$ : $491(\mathrm{M}+\mathrm{Na})^{+}$.

Everlastoside $\mathrm{H}(\mathbf{3})$ : A white powder, $[\alpha]_{\mathrm{D}}^{28}-35.2^{\circ}(c=0.67, \mathrm{MeOH})$ High-resolution positive-ion FAB-MS: Calcd for $\mathrm{C}_{21} \mathrm{H}_{28} \mathrm{O}_{14} \mathrm{Na}(\mathrm{M}+\mathrm{Na})^{+}$ 527.1376; Found 527.1371. UV $\left[\lambda_{\max }(\log \varepsilon), \mathrm{MeOH}\right]: 216$ (4.50), 257 (4.20) nm. IR (KBr, cm $\left.{ }^{-1}\right): 3568,1719,1612,1508,1458,1343,1066 .{ }^{1} \mathrm{H}-$ NMR $\left(500 \mathrm{MHz}, \mathrm{CD}_{3} \mathrm{OD}\right) \delta$ : given in Table $2 .{ }^{13} \mathrm{C}-\mathrm{NMR}(125 \mathrm{MHz}$, $\left.\mathrm{CD}_{3} \mathrm{OD}\right) \delta_{\mathrm{C}}$ : given in Table 2. Positive-ion FAB-MS $m / z: 527(\mathrm{M}+\mathrm{Na})^{+}$

Everlastoside I (4): A white powder, $[\alpha]_{\mathrm{D}}^{23}-32.0^{\circ}(c=0.67, \mathrm{MeOH})$. High-resolution positive-ion FAB-MS: Calcd for $\mathrm{C}_{16} \mathrm{H}_{25} \mathrm{O}_{7}(\mathrm{M}+\mathrm{H})^{+}$ 329.1600; Found 329.1596. IR ( $\left.\mathrm{KBr}, \mathrm{cm}^{-1}\right)$ : 3568, 1509, 1458, 1375, 1071 . ${ }^{1} \mathrm{H}-\mathrm{NMR}\left(500 \mathrm{MHz}, \mathrm{CD}_{3} \mathrm{OD}\right) \delta$ : given in Table $3 .{ }^{13} \mathrm{C}-\mathrm{NMR}(125 \mathrm{MHz}$, $\left.\mathrm{CD}_{3} \mathrm{OD}\right) \delta_{\mathrm{C}}$ : given in Table 3. Positive-ion FAB-MS $m / z: 329(\mathrm{M}+\mathrm{H})^{+}, 351$ $(\mathrm{M}+\mathrm{Na})^{+}$

Everlastoside J (5): A white powder, $[\alpha]_{\mathrm{D}}^{26}-89.3^{\circ}(c=0.30, \mathrm{MeOH})$. High-resolution positive-ion FAB-MS: Calcd for $\mathrm{C}_{21} \mathrm{H}_{32} \mathrm{O}_{11} \mathrm{Na}(\mathrm{M}+\mathrm{Na})^{+}$ 483.1842; Found 483.1846. IR ( $\left.\mathrm{KBr}, \mathrm{cm}^{-1}\right)$ : 3568, 1508, 1458, 1341, 1071. ${ }^{1} \mathrm{H}-\mathrm{NMR}\left(500 \mathrm{MHz}, \mathrm{CD}_{3} \mathrm{OD}\right) \delta$ : given in Table $3 .{ }^{13} \mathrm{C}-\mathrm{NMR}(125 \mathrm{MHz}$, $\left.\mathrm{CD}_{3} \mathrm{OD}\right) \delta_{\mathrm{C}}$ : given in Table 3. Positive-ion FAB-MS $m / z: 461(\mathrm{M}+\mathrm{H})^{+}, 483$ $(\mathrm{M}+\mathrm{Na})^{+}$

Everlastoside $\mathrm{K}(6)$ : A white powder, $[\alpha]_{\mathrm{D}}^{25}-34.2^{\circ}(c=1.04, \mathrm{MeOH})$. High-resolution positive-ion FAB-MS: Calcd for $\mathrm{C}_{22} \mathrm{H}_{34} \mathrm{O}_{12} \mathrm{Na}(\mathrm{M}+\mathrm{Na})^{+}$ 513.1948; Found 513.1937. IR $\left(\mathrm{KBr}, \mathrm{cm}^{-1}\right)$ : $3568,1508,1458,1071 .{ }^{1} \mathrm{H}-$ NMR $\left(500 \mathrm{MHz}, \mathrm{CD}_{3} \mathrm{OD}\right) \delta$ : given in Table $3 .{ }^{13} \mathrm{C}-\mathrm{NMR}(125 \mathrm{MHz}$, $\left.\mathrm{CD}_{3} \mathrm{OD}\right) \delta_{\mathrm{C}}$ : given in Table 3. Positive-ion FAB-MS $m / z$ : $491(\mathrm{M}+\mathrm{H})^{+}, 513$ $(\mathrm{M}+\mathrm{Na})^{+}$

Everlastoside L (7): A white powder, $[\alpha]_{\mathrm{D}}^{22}+34.5^{\circ}(c=0.25, \mathrm{MeOH})$. High-resolution positive-ion FAB-MS: Calcd for $\mathrm{C}_{19} \mathrm{H}_{32} \mathrm{O}_{13} \mathrm{Na}(\mathrm{M}+\mathrm{Na})^{+}$ 525.1373; Found 525.1367. UV [ $\left.\lambda_{\max }(\log \varepsilon), \mathrm{MeOH}\right]: 329$ (4.07) nm. IR $\left(\mathrm{KBr}, \mathrm{cm}^{-1}\right): 3433,1716,1686,1655,1541,1509,1458,1073 .{ }^{1} \mathrm{H}-\mathrm{NMR}$ $\left(500 \mathrm{MHz}, \mathrm{CD}_{3} \mathrm{OD}\right) \delta$ : given in Table $4 .{ }^{13} \mathrm{C}-\mathrm{NMR}\left(125 \mathrm{MHz}, \mathrm{CD}_{3} \mathrm{OD}\right) \delta_{\mathrm{C}}$ : given in Table 4. Positive-ion FAB-MS $m / z: 525(\mathrm{M}+\mathrm{Na})^{+}$

Everlastoside $\mathrm{M}(\mathbf{8})$ : A white powder, $[\alpha]_{\mathrm{D}}^{26}-80.8^{\circ}(c=0.50, \mathrm{MeOH})$. High-resolution positive-ion FAB-MS: Calcd for $\mathrm{C}_{22} \mathrm{H}_{26} \mathrm{O}_{12} \mathrm{Na}(\mathrm{M}+\mathrm{Na})^{+}$ 505.1322; Found 505.1327. UV $\left[\lambda_{\max }(\log \varepsilon), \mathrm{MeOH}\right]: 258(3.87) \mathrm{nm}$. IR $\left(\mathrm{KBr}, \mathrm{cm}^{-1}\right): 3415,1718,1698,1634,1607,1516,1499,1254,1169,1080$. ${ }^{1} \mathrm{H}-\mathrm{NMR}\left(500 \mathrm{MHz}, \mathrm{CD}_{3} \mathrm{OD}\right) \delta$ : given in Table $4 .{ }^{13} \mathrm{C}-\mathrm{NMR}(125 \mathrm{MHz}$, $\left.\mathrm{CD}_{3} \mathrm{OD}\right) \delta_{\mathrm{C}}$ : given in Table 4. Positive-ion FAB-MS m/z: $505(\mathrm{M}+\mathrm{Na})^{+}$

Alkaline and Acid Hydrolysis of Everlastosides F (1), G (2) A solution of 1 or 2 (each $6.0 \mathrm{mg}$ ) in $10 \%$ aqueous $\mathrm{KOH}-50 \%$ aqueous 1,4-dioxane $(1: 1, \mathrm{v} / \mathrm{v}, 1.0 \mathrm{ml})$ was stirred at $37^{\circ} \mathrm{C}$ for $3 \mathrm{~h}$. The reaction mixture was neutralized with Dowex HCR W2 $\left(\mathrm{H}^{+}\right.$form) and the resin was removed by filtration. Evaporation of the solvent from the filtrate under reduced pressure yielded a residue. A part of residue was dissolved in $\left(\mathrm{CH}_{2}\right)_{2} \mathrm{Cl}_{2}(2.0 \mathrm{ml})$ and the solution was treated with $p$-nitrobenzyl $-N-N^{\prime}$-diisopyopylisourea $(10 \mathrm{mg})$, then the whole was stirred at $80^{\circ} \mathrm{C}$ for $1 \mathrm{~h}$. The reaction mixture was subjected to HPLC analysis [column: YMC-Pack ODS-A, $250 \times 4.6 \mathrm{~mm}$ i.d.; mobile phase: $\mathrm{MeOH}-\mathrm{H}_{2} \mathrm{O}(70: 30, \mathrm{v} / \mathrm{v})$; detection: UV $(254 \mathrm{~nm})$; flow rate: $0.9 \mathrm{ml} / \mathrm{min}$ ] to identify the $p$-nitrobenzyl ester of angelic acid $\left(t_{\mathrm{R}}\right.$ $16.0 \mathrm{~min})$, respectively. The rest of residue in $1 \mathrm{M} \mathrm{HCl}(1.0 \mathrm{ml})$ was heated at $80^{\circ} \mathrm{C}$ for $1 \mathrm{~h}$. After cooling, the reaction mixture was neutralized with Amberlite IRA-400 $\left(\mathrm{OH}^{-}\right.$form $)$and then the resin was removed by filtration. Removal of the solvent from the filtrate under reduced pressure, the residue was separated by Sep-Pak C18 cartridge column $\left(\mathrm{H}_{2} \mathrm{O} \rightarrow \mathrm{MeOH}\right)$. The $\mathrm{H}_{2} \mathrm{O}-$ eluted fraction was subjected to HPLC analysis under the following conditions: HPLC column, Kaseisorb $\mathrm{LC} \mathrm{NH}_{2}-60-5,250 \times 4.6 \mathrm{~mm}$ i.d. (Tokyo Kasei Co., Ltd., Tokyo, Japan); detection, optical rotation [Shodex OR-2 (Showa Denko Co., Ltd., Tokyo, Japan); mobile phase, $\mathrm{CH}_{3} \mathrm{CN}-\mathrm{H}_{2} \mathrm{O}$ $(85: 15, \mathrm{v} / \mathrm{v})$; flow rate $0.8 \mathrm{ml} / \mathrm{min}]$. Identification of D-apiose ${ }^{18,22,23)}(\mathbf{i}$, from 2) and D-glucose (ii, from $\mathbf{1}$ and 2) present in the aqueous layer was carried out by comparison of their retention times and optical rotations with those of authentic samples. $t_{\mathrm{R}}$ : (i) $6.6 \mathrm{~min}$ (positive optical rotation) and (ii) $13.9 \mathrm{~min}$ (positive optical rotation), respectively.

Acid Hydrolysis of Everlastosides H (3), I (4), J (5), and K (6) A solution of $3(1.0 \mathrm{mg})$ in $1.0 \mathrm{M} \mathrm{HCl}(1.0 \mathrm{ml})$ was heated at $80^{\circ} \mathrm{C}$ for $3 \mathrm{~h}$. After cooling, the reaction mixture was neutralized with Amberlite IRA-400 $\left(\mathrm{OH}^{-}\right.$ form) and then the resin was removed by filtration. Removal of the solvent from the filtrate under reduced pressure, the residue was separated by Sep- 
Pak C18 cartridge column $\left(\mathrm{H}_{2} \mathrm{O} \rightarrow \mathrm{MeOH}\right)$. The $\mathrm{H}_{2} \mathrm{O}$-eluted fraction was subjected as above to identify D-glucose (ii). Similarly, a solution of 4-6 (each $10.0 \mathrm{mg}$ ) in $1.0 \mathrm{M} \mathrm{HCl}(1.0 \mathrm{ml})$ was heated at $80^{\circ} \mathrm{C}$ for $3 \mathrm{~h}$, and through the similar procedure as above to identify D-apiose (i, from $\mathbf{5}$ ) and Dglucose (ii, from 4-6) from the $\mathrm{H}_{2} \mathrm{O}$-eluted fraction. The $\mathrm{MeOH}$-eluted fraction was purified by HPLC $\left[\mathrm{MeOH}-\mathrm{H}_{2} \mathrm{O}(40: 60, \mathrm{v} / \mathrm{v})\right]$ to give $(2 R, 3 R)$ 1-phenyl-2,3-butandiol ${ }^{26)}$ (4a, 3.0 mg, 59\% from 4, $2.3 \mathrm{mg}, 64 \%$ from 5) and $(2 S, 3 S)$-1-phenyl-2,3-butandiol $\left.{ }^{26}\right)(6 \mathbf{6}, 1.8 \mathrm{mg}, 53 \%$ from $\mathbf{6})$, respectively.

Enzymatic Hydrolysis of Everlastosides H (3) and L (7) A solution of $3(5.8 \mathrm{mg})$ in $\mathrm{H}_{2} \mathrm{O}(2.0 \mathrm{ml})$ was treated with cellulase $(24 \mathrm{mg})$ and the solution was stirred at $37^{\circ} \mathrm{C}$ for $4 \mathrm{~d}$. After EtOH was added to the reaction mixture, the solvent was removed under reduced pressure and the residue was purified by HPLC $\left[\mathrm{MeOH}-\mathrm{H}_{2} \mathrm{O}(30: 70, \mathrm{v} / \mathrm{v})\right]$ to furnish 7-hydroxy-5methoxyphthalide $\left.{ }^{24,25}\right)$ (3a, $\left.1.0 \mathrm{mg}, 50 \%\right)$. Similarly, a solution of $7(2.0 \mathrm{mg})$ in $\mathrm{H}_{2} \mathrm{O}(1.0 \mathrm{ml})$ was treated with tannase $(2.3 \mathrm{mg})$ and the solution was stirred at $37^{\circ} \mathrm{C}$ for $18 \mathrm{~h}$. After EtOH was added to the reaction mixture, the solvent was removed under reduced pressure and the residue was subjected to normal-phase silica gel $\mathrm{CC}\left[500 \mathrm{mg}, \mathrm{CHCl}_{3}-\mathrm{MeOH}-\mathrm{H}_{2} \mathrm{O}\right.$ $(10: 3: 1 \rightarrow 7: 3: 1$, lower layer, $\mathrm{v} / \mathrm{v} / \mathrm{v}) \rightarrow \mathrm{MeOH}]$ to give $4-\left(3^{\prime}\right.$-glucopyranosyloxy-4'-hydroxyphenyl)-3-buten-2-one ${ }^{27)}(7 \mathbf{a}, 0.6 \mathrm{mg}, 44 \%)$ and caffeic $\operatorname{acid}^{28)}(0.3 \mathrm{mg}, 42 \%)$

Acid Hydrolysis of 7 a A solution of $7 \mathbf{a}(0.5 \mathrm{mg})$ in $1.0 \mathrm{M} \mathrm{HCl}(1.0 \mathrm{ml})$ was heated at $80^{\circ} \mathrm{C}$ for $3 \mathrm{~h}$, and through the similar procedure as above to identify D-glucose (ii) from the $\mathrm{H}_{2} \mathrm{O}$-eluted fraction.

Alkaline Hydrolysis of Everlastoside M (8) A solution of $\mathbf{8}(10.0 \mathrm{mg})$ in $10 \%$ aqueous $\mathrm{KOH}-50 \%$ aqueous 1,4 -dioxane $(1: 1, \mathrm{v} / \mathrm{v}, 1.0 \mathrm{ml})$ was stirred at $37^{\circ} \mathrm{C}$ for $5 \mathrm{~h}$. The reaction mixture was neutralized with Dowex $\mathrm{HCR}$ W2 $\left(\mathrm{H}^{+}\right.$form $)$and the resin was removed by filtration. Evaporation of the solvent from the filtrate under reduced pressure yielded a residue, which was subjected to normal-phase silica gel $\mathrm{CC}\left[500 \mathrm{mg}, \mathrm{CHCl}_{3}-\mathrm{MeOH}-\mathrm{H}_{2} \mathrm{O}\right.$ $(10: 3: 1 \rightarrow 7: 3: 1$, lower layer, $\mathrm{v} / \mathrm{v} / \mathrm{v}) \rightarrow \mathrm{MeOH}]$ to give $8 \mathbf{a}(6.7 \mathrm{mg}, 96 \%)$ and $p$-coumaric acid $^{28)}(3.0 \mathrm{mg}, 88 \%)$.

8a: A white powder, $[\alpha]_{\mathrm{D}}^{26}-59.9^{\circ}(c=0.44, \mathrm{MeOH})$. High-resolution positive-ion FAB-MS: Calcd for $\mathrm{C}_{13} \mathrm{H}_{20} \mathrm{O}_{10} \mathrm{Na}(\mathrm{M}+\mathrm{Na})^{+}$359.0954; Found 359.0961. IR ( $\left.\mathrm{KBr}, \mathrm{cm}^{-1}\right): 3786,1718,1655,1509,1458,1073 .{ }^{1} \mathrm{H}-\mathrm{NMR}$ $\left(500 \mathrm{MHz}, \mathrm{CD}_{3} \mathrm{OD}\right) \delta$ : given in Table $4 .{ }^{13} \mathrm{C}-\mathrm{NMR}\left(125 \mathrm{MHz}, \mathrm{CD}_{3} \mathrm{OD}\right) \delta_{\mathrm{C}}$ : given in Table 4. Positive-ion FAB-MS $m / z$ : $359(\mathrm{M}+\mathrm{Na})^{+}$.

Acid Hydrolysis of $8 \mathbf{a}$ A solution of $8 \mathbf{a}(5.0 \mathrm{mg})$ in $1.0 \mathrm{M} \mathrm{HCl}(1.0 \mathrm{ml})$ was heated at $80^{\circ} \mathrm{C}$ for $3 \mathrm{~h}$, and through the similar procedure as above to identify D-glucose (ii) from the $\mathrm{H}_{2} \mathrm{O}$-eluted fraction. The $\mathrm{MeOH}$-eluted fraction was subjected to normal-phase silica gel CC [500 mg, $\mathrm{CHCl}_{3}-\mathrm{MeOH}-\mathrm{H}_{2} \mathrm{O} \quad(7: 3: 1$, lower layer, v/v/v $\left.) \rightarrow \mathrm{MeOH}\right]$ to give (-)shikimic acid ${ }^{28)}(\mathbf{8 b}, 1.6 \mathrm{mg}, 62 \%)$.

Acknowledgements O. M., T. M., and K. N. were supported by a Grantin Aid for Scientific Research from 'High-tech Research Center' Project for Private Universities: matching fund subsidy from The Ministry of Education, Culture, Sports, Science and Technology of Japan (MEXT), 20072011. M. Y., H. M., and S. N. were supported by the 21 st COE Program, Academic Frontier Project, and a Grant-in Aid for Scientific Research from MEXT.

\section{References and Notes}

1) Part XXIX.: Morikawa T., Li X., Nishida E., Nakamura S., Ninomiya K., Matsuda H., Oda Y., Muraoka O., Yoshikawa M., Helv. Chim. Acta, submitted.

2) Yoshikawa M., Sugimoto S., Nakamura S., Matsuda H., Chem. Pharm.
Bull., 55, 571-576 (2007).

3) Yoshikawa M., Xu F., Morikawa T., Pongpiriyadacha Y., Nakamura S., Asao Y., Matsuda H., Chem. Pharm. Bull., 55, 308-316 (2007).

4) Nakamura S., Sugimoto S., Matsuda H., Yoshikawa M., Heterocycles, 71, 577-588 (2007)

5) Yoshikawa M., Nakamura S., Kato Y., Matsuhira K., Matsuda H., Chem. Pharm. Bull., 55, 598-605 (2007).

6) Yoshikawa M., Morikawa T., Asao Y., Fujiwara E., Nakamura S., Matsuda H., Chem. Pharm. Bull., 55, 606-612 (2007)

7) Yoshikawa M., Sugimoto S., Nakmaura S., Sakumae H., Matsuda H., Chem. Pharm. Bull., 55, 1034-1038 (2007)

8) Nakamura S., Sugimoto S., Matsuda H., Yoshikawa M., Chem. Pharm. Bull., 55, 1342-1348 (2007)

9) Yoshikawa M., Wang T., Sugimoto S., Nakamura S., Nagatomo A., Matsuda H., Harima S., Yakugaku Zasshi, 128, 141-151 (2008).

10) Yoshikawa M., Sugimoto S., Kato Y., Nakamura S., Wang T., Yamashita C., Matsuda H., Chem. Biodiversity, 6, 903—915 (2009).

11) Morikawa T., Li X., Nishida E., Ito Y., Matsuda H., Nakamura S., Muraoka O., Yoshikawa M., J. Nat. Prod., 71, 828-835 (2008).

12) Yoshikawa M., Li X., Nishida E., Nakamura S., Matsuda H., Muraoka O., Morikawa T., Chem. Pharm. Bull., 56, 559-568 (2008).

13) Yoshikawa M., Sugimoto S., Nakamura S., Matsuda H., Chem. Pharm. Bull., 56, 1297-1303 (2008).

14) Xie Y., Morikawa T., Ninomiya K., Imura K., Muraoka O., Yuan D., Yoshikawa M., Chem. Pharm. Bull., 56, 1628-1631 (2008).

15) Nakamura S., Okazaki Y., Ninomiya K., Morikawa T., Matsuda H., Yoshikawa M., Chem. Pharm. Bull., 56, 1704-1709 (2008).

16) Sugimoto S., Yoshikawa M., Nakamura S., Matsuda H., Heterocycles, 78, 1023-1029 (2009).

17) Morikawa T., Wang L.-B., Nakamura S., Ninomiya K., Yokoyama E., Matsuda H., Muraoka O., Wu L.-J., Yoshikawa M., Chem. Pharm. Bull., 57, 361-367 (2009).

18) Wang L.-B., Morikawa T., Nakamura S., Ninomiya K., Matsuda H., Muraoka O., Wu L.-J., Yoshikawa M., Heterocycles, 78, 1235-1242 (2009).

19) Ren Z.-Y., Qi H.-Y., Shi Y.-P., Planta Med., 74, 859-863 (2008).

20) Li W., Asada Y., Yoshikawa T., Phytochemistry, 55, 447-456 (2000).

21) The ${ }^{1} \mathrm{H}$ - and ${ }^{13} \mathrm{C}$-NMR spectra of $\mathbf{1}-\mathbf{8}$ and $\mathbf{8 a}$ were assigned with the aid of distortionless enhancement by polarization transfer (DEPT), double quantum filter correlation spectroscopy (DQF COSY), heteronuclear multiple quantum coherence (HMQC), and heteronuclear multiple bond connectivity (HMBC) experiments.

22) The anomeric configurations at D-apiofuranosyl linkages in $\mathbf{2}$ and $\mathbf{5}$ were determined by the comparisons of the ${ }^{13} \mathrm{C}$-NMR data for $\mathbf{2}$ and $\mathbf{5}$ with those for methyl $\alpha$ - and $\beta$-D-apiofuranosides. ${ }^{23)}$

23) Kitagawa I., Sakagami M., Hashiuchi F., Zhou J. L., Yoshikawa M., Ren J., Chem. Pharm. Bull., 37, 551-553 (1989).

24) Opitz L., Hänsel R., Arch. Pharmaz., 304, 228-230 (1971).

25) Satake T., Coskun M., Hori K., Saiki Y., Tanker M., Phytochemistry, 30, 4191-4192 (1991)

26) Awano K., Yanai T., Watanabe I., Takagi Y., Kitahara T., Mori K., Biosci. Biotech. Biochem., 59, 1251-1254 (1995).

27) Ohmura K., Miyase T., Ueno A., Phytochemistry, 28, 1919-1924 (1989).

28) Caffeic acid, $p$-coumaric acid, and (-)-shikimic acid were identified by comparison of their physical and spectral data with those of commercially obtained samples. 